\title{
A Non-WSSUS Channel Simulator for V2X Communication Systems
}

\author{
José Jimmy Jaime-Rodríguez ${ }^{1,+}+\mathbb{C}$, Carlos Antonio Gómez-Vega ${ }^{2,+}+\mathbb{D}$, Carlos A. Gutiérrez ${ }^{2, *,+} \mathbb{(}$, \\ José Martín Luna-Rivera ${ }^{2,+}\left(\mathbb{C}\right.$, Daniel Ulises Campos-Delgado ${ }^{2,+}$ and Ramiro Velázquez $^{3,+}$ \\ 1 Ingeniería Mecatrónica, Unidad Académica Multidisciplinaria Zona Media, \\ Universidad Autónoma de San Luis Potosí. Carretera Rioverde-San Ciro Km 4, \\ El Carmen. San Luis Potosí C.P. 79615, Mexico; jose.jaime@uaslp.mx \\ 2 Facultad de Ciencias, Universidad Autónoma de San Luis Potosí. Av. Chapultepec 1570, \\ Privadas del Pedregal, San Luis Potosí C. P. 78295, Mexico; cgomez@ieee.org (C.A.G.-V.); \\ mlr@fciencias.uaslp.mx (J.M.L.-R.); ducd@fciencias.uaslp.mx (D.U.C.-D.) \\ 3 Facultad de Ingeniería, Universidad Panamericana. Josemaría Escrivá de Balaguer 101, \\ Fracc. Rústicos Calpulli, Aguascalientes C.P. 20290, Mexico; rvelazquez@up.edu.mx \\ * Correspondence: cagutierrez@ieee.org; Tel.: +52-(444)-8-262-300 (ext. 5670) \\ + These authors contributed equally to this work.
}

Received: 19 June 2020; Accepted: 21 July 2020; Published: 24 July 2020

\begin{abstract}
This paper presents a simulator of non-wide sense stationary uncorrelated scattering (non-WSSUS) multipath fading channels for the performance analysis of vehicle-to-everything (V2X) communication systems. The proposed simulator is constructed with the combination of the Monte Carlo and sum-of-cisoids (SOC) principles, and it is suitable for multicarrier transmission schemes such as those defined for dedicated short-range communications (DSRC) and cellular-based V2X (C-V2X) communications. The channel simulator provides an accurate and flexible solution to reproduce the time and frequency (TF) correlation properties of non-WSSUS vehicular channels under arbitrary isotropic and non-isotropic scattering conditions. Furthermore, the proposed simulator allows velocity variations and non-linear trajectories of the mobile stations (MSs). To demonstrate the practical value of the presented simulator, we evaluate the bit error rate (BER) performance of two channel estimation techniques that are considered for IEEE 802.11p transceivers, namely the least squares (LS) estimator and the spectral temporal averaging (STA) technique. The BER performance of both channel estimators was analyzed by considering three propagating scenarios for road safety applications. Our results show that the non-stationary characteristics of the vehicular multipath fading channel have nearly no effects on the LS estimator's BER performance. In contrast, the performance of the STA estimator is significantly affected by the channel's non-stationary characteristics. A variation of the original STA technique that applies only a temporal averaging is introduced in this work to improve the system's BER in non-WSSUS channels.
\end{abstract}

Keywords: channel simulation; dedicated short-range communications; IEEE 802.11p standard; vehicular communications

\section{Introduction}

The worldwide growth of the vehicle fleet has caused high levels of pollution due to inefficient traffic control and increased concerns about the number of deaths and injuries from vehicular accidents. Intelligent transportation systems (ITSs) are a group of technologies that address these problems by managing vehicle operations and assisting drivers with safety and contextual information $[1,2]$. ITSs require a wireless communication platform to keep a vehicle connected with other vehicles and with other elements on the road [3-10]. The technical requirements of this vehicular communication 
platform are more stringent in terms of robustness and latency than those of traditional mobile cellular communications since road safety applications demand a timely transmission and processing of messages that help to bring the driver's awareness to potentially hazardous situations. Such applications aim to decrease the probability of traffic accidents by employing messages that include do-not-pass, emergency-brake, lane change, blind spot, and forward-collision warnings [5]. These warning messages carry information about the position, speed, and acceleration of the transmitting vehicle [4,5], and they are continuously received by neighbor vehicles over a range of hundreds of meters. These safety applications, therefore, rely on direct communication platforms that enable information exchange over short distances.

The dedicated short-range communications (DSRC) systems were the first vehicle-to-everything (V2X) communication technology specifically tailored for ITS applications [3-6]. Both the Federal Communications Commission of the U.S. Government and the European Telecommunications Standards Institute have allocated frequency bands for DSRC operation in the $5.9 \mathrm{GHz}$ spectrum. In addition, the Institute of Electrical and Electronics Engineers (IEEE) developed an amendment to the 802.11 standard for wireless local area networks (WLANs) to address DSRC in vehicular environments within the $5.9 \mathrm{GHz}$ band. This set of specifications for the physical and medium access control layers of DSRC systems, which is known as the IEEE 802.11p standard [11], specifies the use of orthogonal frequency division multiplexing (OFDM) as the transmission scheme for vehicle-to-vehicle (V2V) and vehicle-to-infrastructure (V2I) communications [11]. With these specifications, the V2V and V2I information exchange can be accomplished for relative speeds up to $500 \mathrm{~km} / \mathrm{h}$.

On the other hand, the Third-Generation Partnership Project (3GPP) developed adjustments to the specifications of Long-Term Evolution (LTE) networks to enable V2X applications [8,12]. The device-to-device (D2D) paradigm envisioned in LTE networks supports new use cases related to short-range V2X communications [7-9,13]. Since Release 14, V2X data transmission is supported in LTE devices using two complementary modes: conventional network-based communication for interaction with the cloud, which is known as vehicle-to-network (V2N); and direct D2D communications for high-speed and low-latency V2V, V2I, and vehicle-to-pedestrian (V2P) links [14]. The latter mode is defined for short-range V2X communications employing a direct communications interface (PC5), which specifies the use of single carrier frequency division multiple access (SC-FDMA) as the transmission scheme and operates also in the $5.9 \mathrm{GHz}$ ITS band [14]. Furthermore, 3GPP has also been developing specifications for the upcoming fifth-generation (5G) mobile cellular networks to support V2X communications [15]. This new set of specifications, which started in Release 16, will extend the capabilities of the current LTE-based V2X (LTE-V2X) for new applications, such as autonomous vehicles. In this emerging technology, the $5.9 \mathrm{GHz}$ ITS band is also considered to be exploited for D2D-based V2X applications using the PC5 interface [15].

Despite recent technological and standardization advances, vehicular communications still present important challenges due to the propagation environment and the station's high mobility [16-18]. For this reason, the development of simulation tools that lend themselves to the design, testing, and optimization of V2X communication systems has become a crucial research area. These simulation tools must reproduce the propagation conditions that are observed in vehicular wireless channels to allow the identification of factors that affect the performance of the communication system. Such propagation conditions are simulated following reference channel models that consider particular aspects of the propagation environment, such as multipath interactions, small-scale fading, and shadowing $[16,17]$. Nonetheless, the applicability of the simulation tools should not be restricted to a particular environment. In fact, a flexible channel simulator allows gaining insight into different communication environments and also supports realistic application-related scenarios, e.g., for collision avoidance [16]. The flexibility of the simulator might also enable emulating data transmission under different protocols, such that one can evaluate the system performance considering various sets of specifications for V2X communication technologies. 
A variety of channel simulators has been proposed for vehicular communications. Some of them are based on the wide sense stationary uncorrelated scattering (WSSUS) assumption that is often invoked for the simulation of traditional fixed-to-mobile (F2M) cellular communication channels [19-21]. However, recent empirical findings have demonstrated that the WSSUS condition [22] is not satisfied in vehicular environments [23-25]. For this reason, several non-WSSUS V2V channel simulators have also been developed in the literature [26-29]. Some of these non-WSSUS channel simulators take as input the geometric structure and physical properties of the propagation environment and follow the principles of ray tracing simulation to compute the channel impulse response (CIR). These simulators provide a good approximation to the response of real-world channels, but they require a high amount of computational resources to reproduce the propagation conditions by considering the physical properties and the location of interfering objects (IOs). Therefore, these tools are not easily scalable to support system-level simulations that allow the evaluation of the system's performance. Time-delay filter models are another approach to simulate the vehicular channel [27-29]. In this approach, both the amplitude and phase of each filter coefficient are modeled using a correlation matrix. Nonetheless, these simulators are not straightforward to reconfigure to different scenarios of interest.

This paper presents a non-WSSUS V2X channel simulator for the performance evaluation of V2X communication systems. Such a simulator is based on the combination of the Monte Carlo and sum-of-cisoids (SOC) principles [30]. This tool is intended for V2X communication systems based on multicarrier techniques where the information is transmitted in frames, such as the DSRC and cellular-based V2X (C-V2X) systems. The simulator is based on a reference V2X channel model that allows velocity variations and non-linear trajectories of the mobile stations (MSs) [31]. Furthermore, this reference model provides information about the time and frequency (TF) correlation properties of non-WSSUS channels under arbitrary isotropic and non-isotropic scattering conditions. To exemplify the practical value of the proposed simulator, we present a bit error rate (BER) performance analysis of two channel estimation techniques that are considered for IEEE 802.11p transceivers: the least squares (LS) estimator, and the spectral temporal averaging (STA) technique [32]. Our results show that the performance of the LS estimator decreases whenever the relative speed of the MSs or the frame length increases. These performance degradations are due to the inability of the LS estimator to dynamically track the channel variations in the time domain. On the other hand, the performance of the STA technique is in general poor regardless of the relative speed, acceleration, frame length, or scattering conditions since the frequency-domain averaging operations are not effective in compensating the channel's non-stationary characteristics. Therefore, we introduce a modification to the original STA technique that considers only the temporal averaging. This modification aims to improve the BER performance of the system in non-WSSUS channels and overcomes the challenge of equalizing large data frames, which is a drawback of the LS estimator. In general, our results show the applicability of the proposed simulator to evaluate the performance of V2X systems under different propagation conditions in road safety scenarios.

The rest of this paper is organized as follows. In Section 2, we expand the discussion of wireless communications in road safety scenarios and of the state-of-the-art in vehicular channel simulators. In Section 3, we review the specifications for V2X communication systems given in the IEEE 802.11p and the LTE-V2X standards. In that section, we also present a mathematical model of a multicarrier data frame that is valid for both the IEEE 802.11p and the LTE-V2X standards. In Section 4, we present the reference channel model. Then, in Section 5, we introduce the simulation model for the described reference channel. In Section 6, we describe the IEEE 802.11p estimation techniques and present the simulation results in three road safety scenarios. Finally, we present the conclusions of this research work in Section 7.

Notation: Capital bold letters denote matrices. Lowercase bold letters correspond to vectors. The scalar element placed in the $m$-th row and the $k$-th column of a matrix $\mathbf{M}$ is represented by $\langle\mathbf{M}\rangle_{m, k}$. The set of the complex numbers is denoted as $\mathbb{C}$. 


\section{Motivation and Related Work}

The work presented in this paper is motivated by the need for a flexible vehicular channel simulator that enables the emulation of propagation scenarios similar to those found in road safety applications [1,2]. These scenarios are characterized, on the one hand, by a small coverage area, according to the distance covered by vehicles during the transmission of warning messages, and on the other hand, by the vehicles' high mobility, which may include speed variations and changes in the vehicles' trajectories [3-5]. The relevance of a simulation framework with these capabilities lies in the fact that most of the existing vehicular channel simulators do not incorporate the aforementioned properties. The early work in modeling and simulation of vehicular channels assumed: (i) the uniform motion of the vehicles, (ii) the transmission of narrowband signals, and (iii) the fulfillment of the wide-sense stationary (WSS) condition, e.g., [33-36]. Subsequent work was focused on extending these initial efforts with respect to small-scale wideband channels by assuming WSS statistical properties in the time and frequency domains, i.e., with respect to locally WSSUS vehicular channels [37-39]. Nonetheless, empirical evidence showed that the vehicular radio channels do not fulfill the WSSUS condition [23-25], and this issue recently motivated a further wave of research initiatives aiming at the formulation of new statistical reference models for the simulation of locally non-WSSUS wideband channels. Contributions in this regard include the work presented in [40-44].

Most of the papers dealing with the modeling of locally non-stationary vehicular channels, either narrowband or wideband, assume uniform motion of the MSs, that is vehicles moving on a linear trajectory and with a constant speed. To the best of the authors' knowledge, Iqbal and Abhayapala were the first to formulate a non-stationary mobile radio channel model that considers the effects of non-uniform motion in an F2M wireless link, where the MS is moving with a constant acceleration on a linear trajectory [45]. This F2M channel model was extended by Pätzold and Borhani in [46] with respect to non-linear trajectories. The proposals in [46] were applied by Dahech et al. [47] to characterize non-stationary V2X channels allowing for speed and trajectory variations of the vehicles [47]. Based on the concepts discussed in [45-47], novel simulation models for non-stationary vehicular channels under velocity variations of the MSs were proposed independently in [48,49]. In the aforementioned papers, the spotlight was on narrowband channels. In [31], Gutiérrez et al. proposed a statistical model of locally non-stationary wideband V2X channels that incorporates the effects of speed and trajectory variations. The scope of the latter paper was limited to the formulation and statistical analysis of the channel model. As an extension, in this paper, we provide a methodology for the simulation of the channel model in [31]. The simulation framework presented here was conceived of as a tool for the performance analysis of vehicular communication systems based on multicarrier modulation techniques, such as the DSRC and C-V2X systems reviewed next.

\section{Multicarrier Data Frames of the DSRC and LTE-V2X Standards}

In this section, we present a brief overview of the IEEE 802.11p and LTE-V2X specifications for the physical layer of vehicular communication systems. In the case of the LTE-V2X, we review the characteristics of the PC5 interface, which is considered for V2V, V2I, and V2P applications. Specifically, the parameters of the multicarrier schemes for both sets of specifications, as well as their frame structures are presented for a typical $10 \mathrm{MHz}$ bandwidth. Then, we describe a mathematical reference model of the received multicarrier data frame that is valid for both sets of specifications.

\subsection{General Overview of IEEE 802.11p and LTE-V2X Specifications}

The IEEE $802.11 \mathrm{p}$ standard was developed as an amendment to the specifications for WLANs to address wireless communication in vehicular environments $[4,5]$. This standard presents the specifications for DSRC systems in the $5.9 \mathrm{GHz}$ band. The IEEE 802.11p transceivers use OFDM as a modulation technique, in which a block of data is transmitted through equidistant subcarriers. The data transmission can be performed over three different bandwidths, which can be 5, 10, or $20 \mathrm{MHz}$. 
The preferred bandwidth is $10 \mathrm{MHz}$ since it is more robust against the time dispersion of the transmitted signal [4]. The IEEE $802.11 \mathrm{p}$ standard considers a total of 64 subcarriers, among which 48 are used for data transmission and four are used as pilot subcarriers. The remaining 12 subcarriers do not transmit information to avoid interference between adjacent bands. The parameters of an IEEE 802.11p OFDM symbol for a $10 \mathrm{MHz}$ bandwidth are shown in Table 1 [11]. Furthermore, the frame structure considers short training symbols (STSs) with a duration of $1.6 \mu$ s and long training symbols (LTSs) of $6.4 \mu \mathrm{s}$. In addition, the specifications consider a guard interval (GI) of $1.6 \mu \mathrm{s}$ to reduce intersymbol interference. Figure 1 shows the frame structure for a typical $10 \mathrm{MHz}$ bandwidth, as specified in the IEEE 802.11p. First, ten STSs $\left(t_{1}, \ldots, t_{10}\right)$ are considered as a preamble for signal detection, automatic gain control, and diversity selection $\left(t_{1}, \ldots, t_{7}\right)$, as well as for coarse frequency offset estimation and timing synchronization $\left(t_{8}, t_{9}\right.$, and $\left.t_{10}\right)$. Next, there are two LTSs $\left(T_{1}\right.$ and $\left.T_{2}\right)$ that follow two GIs, which are placed after the tasks in the preamble. The LTSs $T_{1}$ and $T_{2}$ are employed for channel estimation and fine frequency offset estimation. Then, the frame considers a header that consists of a GI and a signal block where the bit rate and frame length are defined. Finally, the frame ends with the the data field, which includes one GI of $1.6 \mu$ s for each information block. The IEEE 802.11 standard does not restrict the frame length, but it recommends a size between one and 4096 bytes according to the application requirements [11].

Table 1. OFDM symbol parameters for IEEE 802.11p and LTE-V2X with a $10 \mathrm{MHz}$ bandwidth [11,14].

\begin{tabular}{ccc}
\hline Parameter & IEEE 802.11p & LTE-V2X \\
\hline Data subcarriers $\left(N_{p d}\right)$ & 48 & 600 \\
\hline Pilot $\left(N_{p p}\right)$ & 4 subcarriers & 4 symbols \\
\hline Frequency spacing between subcarriers $(\Delta f)$ & $156.25 \mathrm{kHz}$ & $15 \mathrm{kHz}$ \\
\hline Guard interval & $1.6 \mu \mathrm{s}$ & $4.69 \mu \mathrm{s}$ \\
\hline Symbol duration without Guard interval & $6.4 \mu \mathrm{s}$ & $66.31 \mu \mathrm{s}$ \\
\hline Total symbol duration $\left(T_{s}\right)$ & $8 \mu \mathrm{s}$ & $71 \mu \mathrm{s}$ \\
\hline
\end{tabular}

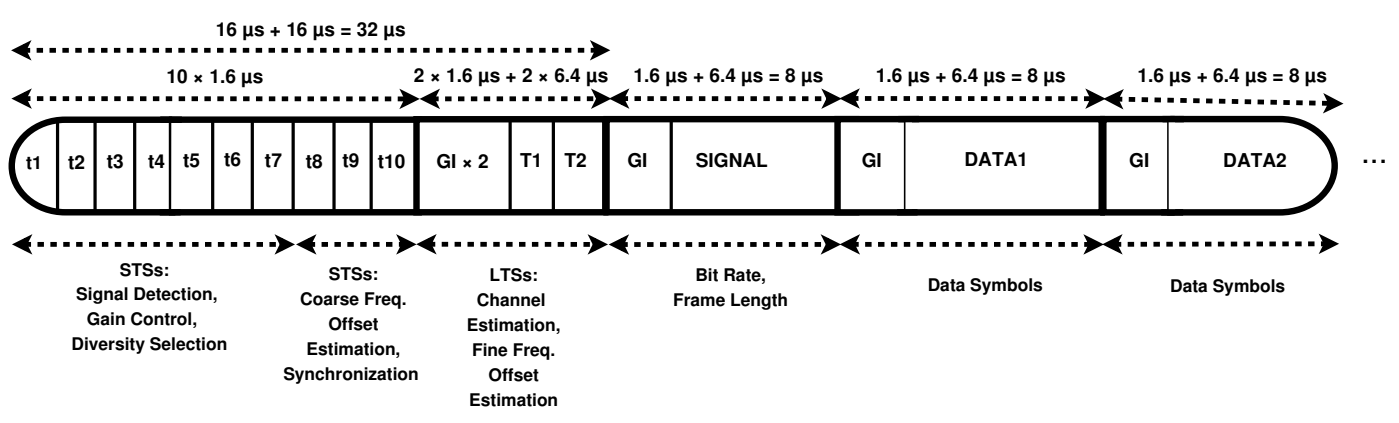

Figure 1. IEEE 802.11p frame structure with a $10 \mathrm{MHz}$ bandwidth [11].

With respect to LTE-V2X, 3GPP included a set of specifications to the LTE standard for applications in vehicular environments [14]. Two operation modes are defined in such 3GPP specifications. The first mode allows vehicles to communicate with the cellular network for cloud-based applications, whereas the second mode enables V2X short-range communications. This latter mode is based on the PC5 interface that is considered for direct D2D communications [14]. Such a mode is referred to as sidelink transmission [9]. The PC5 interface operates in the $5.9 \mathrm{GHz}$ band using SC-FDMA as the transmission scheme. The specifications of the PC5 interface consider resource blocks (RBs) that consist of 12 subcarriers. Depending on the bandwidth, the transmission scheme is scalable by carrier aggregation, which adds new resource blocks to the symbol. For a typical $10 \mathrm{MHz}$ bandwidth, the specifications define 50 RBs, which occupy a total of 600 data subcarriers [14]. The main parameters of LTE-V2X employing the PC5 interface for a $10 \mathrm{MHz}$ bandwidth are shown in 
Table 1 [14]. The subcarrier spacing is set to $15 \mathrm{kHz}$ (i.e., $180 \mathrm{kHz}$ per RB). Additionally, the frame duration is fixed to $1 \mathrm{~ms}$, which allows the transmission of 14 symbols including GIs. The duration of each symbol and GI is $66.31 \mu \mathrm{s}$ and $4.69 \mu \mathrm{s}$, respectively. The frame structure is shown in Figure 2, where the symbols are enumerated from 0 to 13. The LTE-V2X frame includes nine data symbols, four demodulation reference signal (DMRS) symbols and one empty symbol for $T_{X}-R_{X}$ switch and timing adjustment $[9,14]$. The last symbol allows tracking the channel for the next transmission. These parameters are likely to be employed by emerging C-V2X technologies since $5 \mathrm{G}$ specifications are also considering the use of the PC5 interface for direct D2D applications in vehicular environments [15].

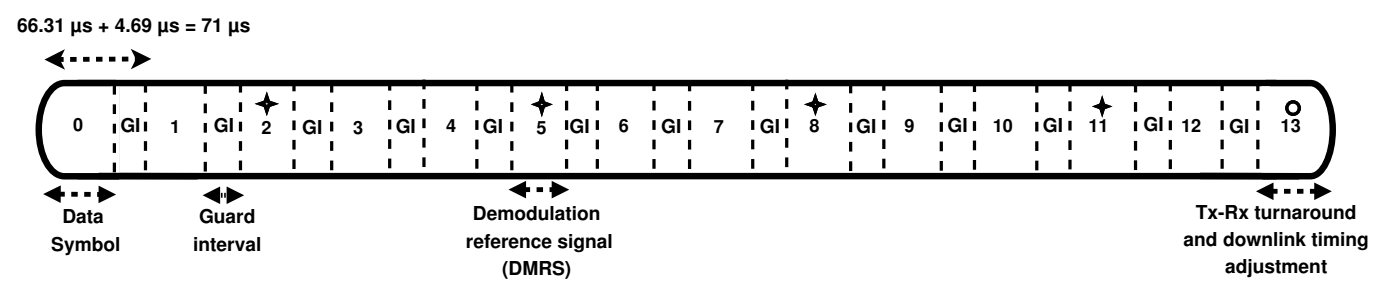

Figure 2. LTE-V2X frame structure with a $10 \mathrm{MHz}$ bandwidth [14].

\subsection{Mathematical Model of the Received Signal in Multicarrier Systems}

The channel simulator proposed in this paper is based on a signal model that is suitable for multicarrier communications that transmit the information employing data frames. This signal model is valid for the data frames specified by the IEEE 802.11p and 3GPP LTE-V2X standards. We consider $N_{p}$ subcarriers for data transmission. Therefore, we model the transmitted symbols in the complex baseband equivalent as column vectors of $N_{p}$ elements where each element corresponds to the data in the $n$-th subcarrier. Then, considering a frame composed of $K$ multicarrier (OFDM) symbols, the transmitted signal is described by an $N_{p} \times K$ matrix $\mathbf{X} \in \mathbb{C}^{N_{p} \times K}$.

Considering the transmission of the data frame, the received signal can be expressed as:

$$
\mathbf{Y}=\mathbf{H} \circ \mathbf{X}+\mathbf{N}
$$

where $\mathbf{Y}, \mathbf{H}$, and $\mathbf{N} \in \mathbb{C}^{N_{p} \times K}$ represent respectively the received symbols matrix, the channel transfer function matrix, and the additive white Gaussian noise (AWGN) matrix. Furthermore, the operator $\circ$ represents the Hadamard product.

\section{Reference Channel Model}

In this section, we describe the reference channel model that assumes non-linear trajectories and velocity variations of the MSs. We introduce first the reference propagation environment at the beginning of the transmission. Then, we present the channel's transfer function and describe its parameters under the one-ring scattering assumption.

\subsection{Description of the Reference Propagation Environment}

The proposed channel simulator in this work is based on the reference model for vehicular communications presented in [31]. Such a model considers velocity variations and non-linear trajectories of the MSs. This model is particularly well suited for analyzing the channel's non-stationarities arising at a small-scale level due to the time-varying nature of the propagation delays [42] and the frequency dependence of the Doppler shift [50]. In this model, the transmitted signal reaches the receiving antenna by single interactions with $\mathcal{L}$ fixed (static) IOs randomly located in the propagation environment. As a result of such interactions, $\mathcal{L}$ replicas of the transmitted signal are observed at the receiving antenna.

Figure 3 shows the reference propagation environment when the MSs begin the transmission at $t=0$. The black dots represent the IOs, whereas the black triangle and the black square denote the transmitter $\left(T_{X}\right)$ and receiver $\left(R_{X}\right)$ stations, respectively. The initial positions of $T_{X}$ and $R_{X}$ are represented 
by $\mathcal{O}$ y $\mathcal{O}^{\prime}$, respectively, and they are separated by a distance $D$. Vectors $\mathbf{v}_{q}$ and $\mathbf{a}_{q}$ for $q \in\{T, R\}$ represent the velocity and constant acceleration components, respectively, of $T_{X}$ and $R_{X}$. The velocity vectors define the initial speeds and directions of motion of the MSs. The initial speeds of $T_{X}$ and $R_{X}$ are, respectively, $v_{T}$ and $v_{R}$. The angles $\gamma_{T}$ and $\gamma_{R}$ correspond to the directions of the velocity components of $T_{X}$ and $R_{X}$, respectively. On the other hand, the acceleration components define the magnitudes and directions for the MS changes of motion over time. The acceleration magnitudes of $T_{X}$ and $R_{X}$ are $a_{T}$ and $a_{R}$, respectively. The directions of the acceleration vectors are $\rho_{T}$ and $\rho_{R}$ for $T_{X}$ and $R_{X}$, respectively. Furthermore, $\mathbf{p}_{\ell}^{T}$ denotes the position of the $\ell$-th IO with respect to the reference point $\mathcal{O}$, and $\mathbf{p}_{\ell}^{\mathbf{R}}$ describes the $R_{X}$ position seen from the $\ell$-th IO. The $\ell$-th IO is separated by a distance $d_{\ell}^{T}$ from $T_{X}$ and a distance $d_{\ell}^{R}$ from $R_{X}$. Finally, $\phi_{\ell}^{T}$ represents the angle of departure (AOD) and $\phi_{\ell}^{R}$ the angle of arrival (AOA), both of them from the interaction with the $\ell$-th IO. The vectors introduced in this section, as well as the constants that complete the description of the propagation environment are summarized in Table 2, where the notation $\mathbf{x}=M_{x} \angle \Theta_{x}$ indicates a vector $\mathbf{x}$ with magnitude $M_{x}$ and direction $\Theta_{x}$.

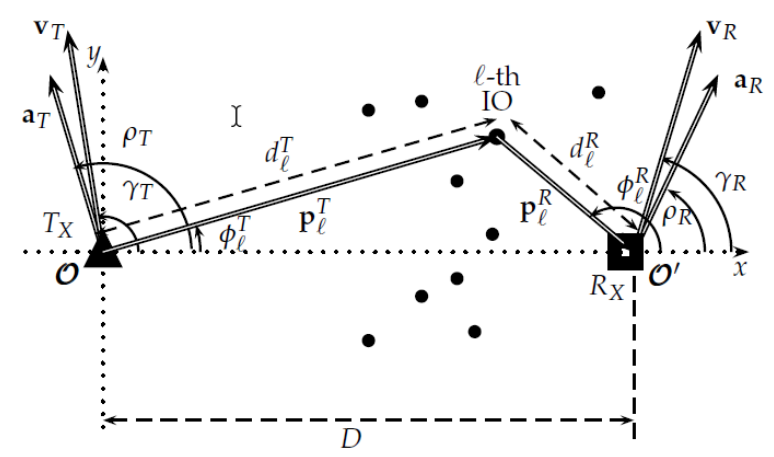

Figure 3. Reference propagation environment at $t_{0}=0$.

Table 2. List of parameters that describe the propagation scenario $\left(\mathbf{x}=M_{x} \angle \Theta_{x}\right.$ denotes a vector $\mathbf{x}$ with magnitude $M_{x}$ and direction $\Theta_{x}$ ).

\begin{tabular}{lc}
\hline Description & Parameter \\
\hline Velocity vector of $T_{X}$ & $\mathbf{v}_{T}=v_{T} \angle \gamma_{T}$ \\
\hline Velocity vector of $R_{X}$ & $\mathbf{v}_{R}=v_{R} \angle \gamma_{R}$ \\
\hline Acceleration vector of $T_{X}$ & $\mathbf{a}_{T}=a_{T} \angle \rho_{T}$ \\
\hline Acceleration vector of $R_{X}$ & $\mathbf{a}_{R}=a_{R} \angle \rho_{R}$ \\
\hline Position of the $\ell$-th IO with respect to the reference point $\mathcal{O}$ & $\mathbf{p}_{\ell}^{T}=d_{\ell}^{T} \angle \phi_{\ell}^{T}$ \\
\hline$R_{X}$ position seen from the $\ell$-th IO & $\mathbf{p}_{\ell}^{R}=d_{\ell}^{R} \angle \phi_{\ell}^{R}$ \\
\hline Initial distance between $T_{X}$ and $R_{X}$ & $D$ \\
\hline Radius of the ring of IOs around $R_{X}$ & $d$ \\
\hline Mean of the AOA distribution & $\mu_{R}$ \\
\hline Concentration parameter of the AOA distribution & $\kappa$ \\
\hline
\end{tabular}

\subsection{Mathematical Model of the Channel Transfer Function}

Following [31], the transfer function for non-WSSUS channels with Rayleigh fadings is represented as follows:

$$
H[m, k]=\Pi_{T_{0}}[k] \sum_{\ell=1}^{\mathcal{L}} g_{\ell} \times \exp \left\{-j\left(\theta_{\ell}+2 \pi \tau_{\ell}[k]\left(f_{c}+m \Delta f\right)\right)\right\}
$$


where $j^{2}=-1, m$ corresponds to the frequency subcarrier index, $k$ is the data symbol index, $\Delta f$ denotes the separation between subcarriers, $\mathcal{L}$ represents the total number of IOs around $R_{X}, g_{\ell}$ and $\tau_{\ell}[k]$ are the amplitude and the time-varying propagation delay of the $\ell$-th replica of the transmitted signal, and $\theta_{\ell}$ stands for the phase shift caused by the interaction of the transmitted signal with the $\ell$-th IO. The carrier frequency is given by $f_{c}=c / \lambda$, with $c$ denoting the speed of light and $\lambda$ the wavelength of the transmitted signal. Furthermore, $\Pi_{T_{0}}[k]$ is defined as:

$$
\Pi_{T_{0}}[k] \triangleq \begin{cases}1, & 0 \leq k T_{s} \leq T_{0} \\ 0, & \text { others }\end{cases}
$$

where $T_{S}$ is the duration of a single symbol. The function $\Pi_{T_{0}}[k]$ denotes a rectangular windowing function introduced to constrain $H[m, k]$ within a time interval $T_{0}$ where the plane wave approach is valid.

The gains $g_{\ell}$ are assumed positive random variables, whereas the phase shifts $\theta_{\ell}$ are independent and identically distributed (i.i.d.) random variables with a circular uniform distribution in $[-\pi, \pi)$. The time-varying propagation delays $\tau_{\ell}[k]$ in (2) are defined by:

$$
\tau_{\ell}[k]=\frac{d_{\ell}^{T}+d_{\ell}^{R}}{c}-\frac{k T_{S}}{f_{c}}\left[f_{\ell}^{S}+\frac{\dot{f}_{\ell}^{A}[k]}{2}\right]
$$

where $f_{\ell}^{S}$ and $\dot{f}_{\ell}^{A}[k]$ characterize the Doppler frequency shifts caused by the initial speed and the acceleration of the MSs, respectively, and they are given by:

$$
\begin{gathered}
f_{\ell}^{S}=f_{\max }^{T} \cos \left(\phi_{\ell}^{T}-\gamma_{T}\right)+f_{\max }^{R} \cos \left(\phi_{\ell}^{R}-\gamma_{R}\right) \\
\dot{f}_{\ell}^{A}[k]=\dot{f}_{\max }^{T} \cos \left(\phi_{\ell}^{T}-\rho_{T}\right)+\dot{f}_{\max }^{R} \cos \left(\phi_{\ell}^{R}-\rho_{R}\right)
\end{gathered}
$$

with $f_{\max }^{q}=v_{q} / \lambda$ and $\dot{f}_{\max }^{q}[k]=\left(k T_{s} a_{q}\right) / \lambda$ for $q \in\{T, R\}$, which correspond to the maximum Doppler frequency shifts due to the initial speed and the acceleration of $T_{X}$ and $R_{X}$, respectively. The parameters $v_{q}$ and $a_{q}$ for $q \in\{T, R\}$ represent the initial speeds and accelerations of the MSs. Variables $\gamma_{q}$ and $\rho_{q}$ for $q \in\{T, R\}$ are deterministic quantities that characterize the initial direction of motion of the MSs and the direction of their acceleration components, respectively.

The AODs and AOAs are circular symmetric random variables having distributions $p_{\phi}^{T}(\phi)$ and $p_{\phi}^{R}(\phi)$, respectively. We consider that the function $p_{\phi}^{R}(\phi)$ follows a von Mises distribution given by [51]:

$$
p_{\phi}^{R}\left(\phi ; \mu_{R}, \kappa\right)=\frac{e^{\kappa \cos \left(\phi-\mu_{R}\right)}}{2 \pi I_{0}(\kappa)}
$$

where $\mu_{R}$ is the mean of the distribution, $\kappa$ is known as the concentration parameter, and $I_{0}(\kappa)$ is the modified zeroth order Bessel function of the first kind [51]. When $\kappa$ increases its value, the values of $\phi_{\ell}^{R}$ accumulate around its mean value. A particular case of this function is when $\kappa=0$. In this case, the values $\phi_{\ell}^{R}$ follow a uniform distribution over $[-\pi, \pi)$. The von Mises distribution in (7) allows modeling arbitrary propagation scenarios with isotropic scattering $(\kappa=0)$ and non-isotropic scattering $(\kappa>0)$.

Considering that only one side of the radio link is affected by local interactions with IOs and that such interactions occur in the receiver side, the AODs $\phi_{\ell}^{T}$ can be defined as [31]:

$$
\phi_{\ell}^{T}=\arctan \left(\frac{d_{\ell}^{R} \sin \left(\phi_{\ell}^{R}\right)}{D+d_{\ell}^{R} \cos \left(\phi_{\ell}^{R}\right)}\right)
$$


where $d_{\ell}^{T}$ and $d_{\ell}^{R}$ depend on $\phi_{\ell}^{R}$ and $\phi_{\ell}^{R}$ as follows:

$$
\begin{aligned}
& d_{\ell}^{T}=\mathcal{G}_{T}\left(\phi_{\ell}^{T}\right)=\sqrt{D^{2}+d^{2}-2 d D \cos \left(\phi_{\ell}^{R}\right)} \\
& d_{\ell}^{R}=\mathcal{G}_{R}\left(\phi_{\ell}^{R}\right)=d
\end{aligned}
$$

when the IOs are assumed to be located on a ring of radius $d$ centered on $\mathcal{O}^{\prime}$.

\section{The Proposed Vehicular Channel Simulator}

For the simulation of the reference channel model given by the transfer function $H[m, k]$ in (2), we follow the principle of deterministic channel modeling [52]. According to this principle, the simulation of a non-realizable stochastic reference channel model can be accomplished by generating a single realization or sample function in each simulation run. Therefore, a realization of the reference channel can be computed by following the transfer function $[53,54]$ :

$$
\widetilde{H}[m, k]=\Pi_{T_{0}}[k] \sum_{\ell=1}^{\mathcal{L}} \widetilde{g}_{\ell} \times \exp \left\{-j\left(\widetilde{\theta}_{\ell}+2 \pi \widetilde{\tau}_{\ell}[k]\left(f_{c}+m \Delta f\right)\right)\right\}
$$

where $\widetilde{g_{\ell}}=\sqrt{\frac{1}{\mathcal{L}}}$ for all $\ell$ is an observation of the amplitude, $\widetilde{\theta}_{\ell} \in[-\pi, \pi)$ is a single realization of the phase shift that follows a uniform circular distribution, and the time-varying propagation delays $\widetilde{\tau}_{\ell}[k]$ are given by:

$$
\widetilde{\tau}_{\ell}[k]=\frac{\mathcal{G}_{T}\left(\widetilde{\phi}_{\ell}^{T}\right)+\mathcal{G}_{R}\left(\widetilde{\phi}_{\ell}^{R}\right)}{C}-\frac{k T_{S}}{f_{c}}\left[\widetilde{f}_{\ell}^{S}+\frac{\tilde{\hat{f}}_{\ell}^{A}[k]}{2}\right]
$$

with:

$$
\begin{gathered}
\widetilde{f}_{\ell}^{S}=f_{\max }^{T} \cos \left(\widetilde{\phi}_{\ell}^{T}-\gamma_{T}\right)+f_{\max }^{R} \cos \left(\widetilde{\phi}_{\ell}^{R}-\gamma_{R}\right) \\
\tilde{f}_{\ell}^{A}[k]=\dot{f}_{\max }^{T} \cos \left(\widetilde{\phi}_{\ell}^{T}-\rho_{T}\right)+\dot{f}_{\max }^{R} \cos \left(\widetilde{\phi}_{\ell}^{R}-\rho_{R}\right)
\end{gathered}
$$

where $\widetilde{\phi}_{\ell}^{T}, \widetilde{\phi}_{\ell}^{R} \in[-\pi, \pi)$ are realizations of the AOD and AOA, respectively, that follow the distributions of the circular random variables $\phi_{\ell}^{T}$ and $\phi_{\ell}^{R}$, as described in Section 4.2. The rest of the parameters $\mathcal{L}, f_{c}, D, d, v_{q}, a_{q}, \gamma_{q}$, and $\rho_{q}$ for $q \in\{T, R\}$, as well as $\mu_{R}$ and $\kappa$ for the distribution of $\phi_{\ell}^{R}$ are deterministic quantities introduced by the user in order to generate arbitrary propagation environments. This simulation model can be classified as an SOC model of Class XII according to the classification described in [55].

The described channel simulator is suitable for the performance analysis of V2X communication systems based on multicarrier transmission schemes, such as specified in the current $\mathrm{V} 2 \mathrm{X}$ communication standards. For this purpose, we consider that the system uses $N_{p}$ subcarriers to transmit $K$ symbols. Then, the simulation model in (11) can be used as $\langle\widetilde{\mathbf{H}}\rangle_{m, k}=\widetilde{H}\left[m-N_{p} / 2, k-1\right]$ for $m=1, \ldots, N_{p}$ and $k=1, \ldots, K$ to generate the channel transfer function matrix. This expression allows us to start the transmission at time $t=0$ and generate a spectrum centered at the carrier frequency $f_{c}$. A realization of the channel model requires us to specify the bandwidth $B$ and the carrier frequency $f_{c}$, as well as the spacing in time and frequency according to the system specifications (e.g., IEEE 802.11p or LTE-V2X) for a total of $K$ symbols and $N_{p}$ subcarriers. Furthermore, the propagation scenario should be initialized by specifying the number of IOs, the scattering parameters, and the components for the dynamics of the MSs. Besides, for the plane wave propagation approach to be valid in the simulation channel model, the far-field condition must be met. Therefore, we consider defining arbitrary distance values such that $D \gg d$. Table 3 shows the pseudocode for the function GenerateChannel, which produces a single realization of the channel based on the proposed simulation model. This function takes as input the motion parameters for the MSs $\left(v_{T}, v_{R}, a_{T}, a_{R}, \gamma_{T}, \gamma_{R}, \rho_{T}, \rho_{R}\right)$, the number of IOs $\mathcal{L}$, the scattering parameters $\left(\mu_{R}\right.$ and $\left.\kappa\right)$, the distance between MSs $D$, the radius $d$ of the ring of IOs, and the number of total symbols $K$. We consider that the 
system parameters are defined inside the function following the desired V2X specifications. First, we set all the system parameters according to the V2X standard for a bandwidth $B$. Then, we generate the corresponding random variables and compute the Doppler shifts following the simulation model with the user inputs for an arbitrary propagation scenario. Finally, a realization of the channel transfer function is computed and returned to simulate data transmission.

Table 3. Pseudocode of the proposed channel simulator.

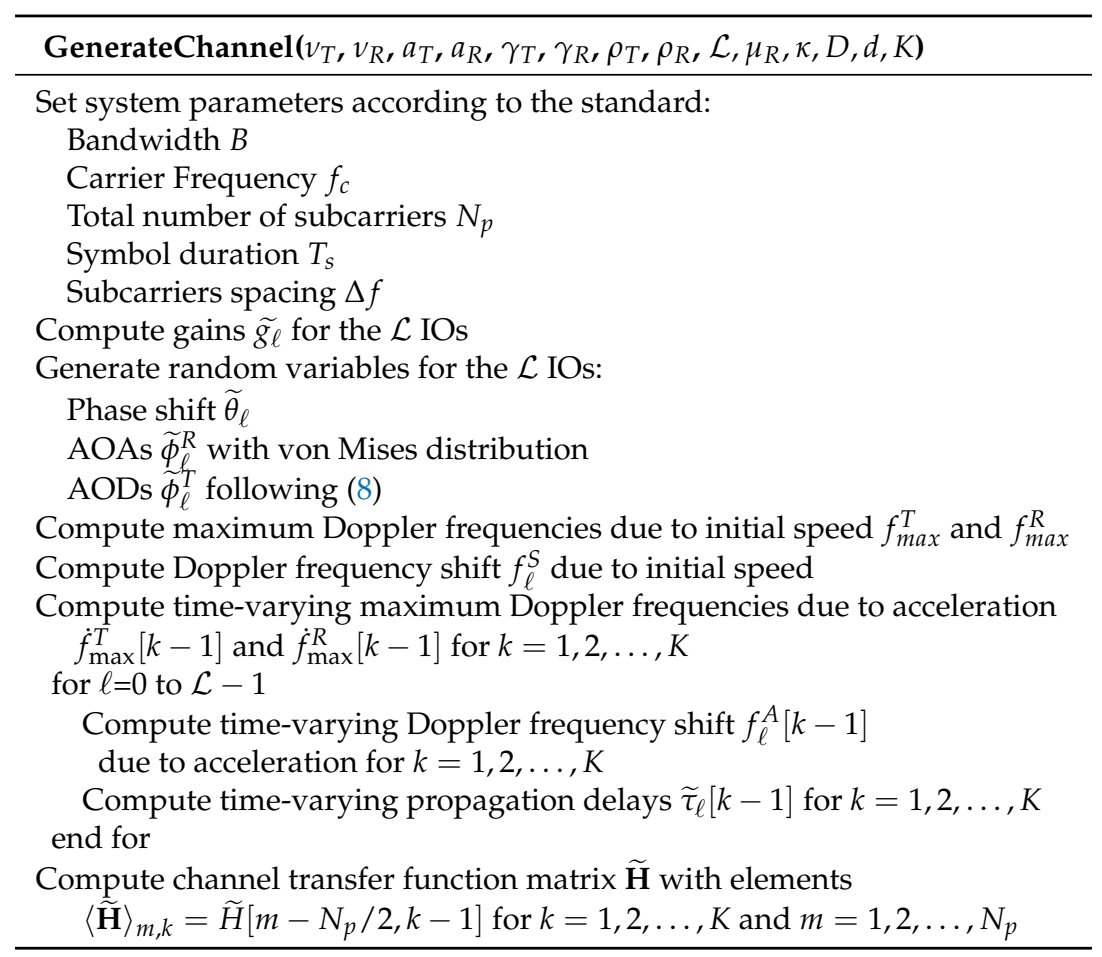

The simulation of a V2X communication system requires several blocks in addition to the channel simulator. These blocks realize different communication tasks such as modulation, equalization, and demodulation. Figure 4 shows a block diagram for the simulation of a V2X communication system, which consists of three main blocks: the transmitter, the wireless propagation channel, and the receiver. First, in the transmitter side, the training and data generator are used to obtain random information frames to be transmitted. We consider that $K$ symbols are generated with $N_{p}$ subcarriers. Then, the information symbols are modulated, i.e., the information is encoded from the message source in a way that is suitable for transmission. Next, the modulated signal is sent through the multiplicative non-WSSUS V2X channel that is also corrupted by AWGN. The V2X channel in this block diagram is obtained using the GenerateChannel function with an arbitrary propagation scenario. In the receiver side, we consider channel estimation and equalization blocks to mitigate the impact of the wireless channel. Finally, the signal is demodulated to decode the message information.

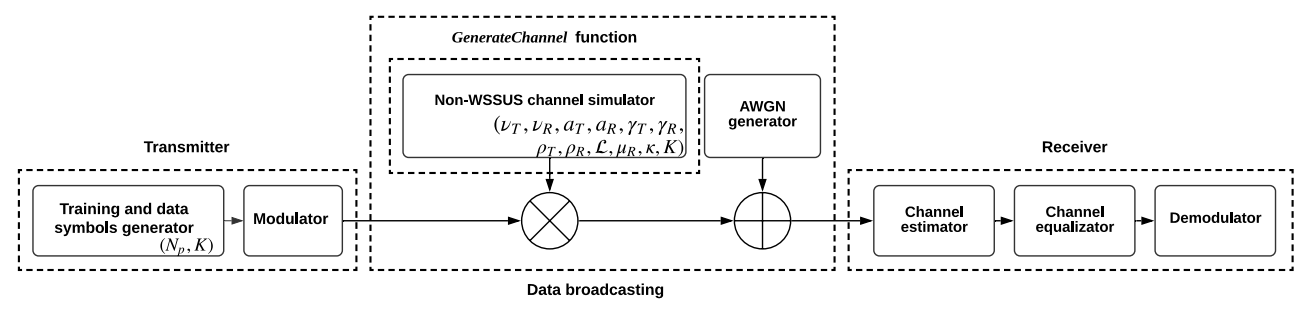

Figure 4. Block diagram for the simulation of a general V2X communication system. 
Table 4 shows the pseudocode to simulate a V2X communication system to evaluate its performance in terms of the BER. The implementation should consider a set of specifications (e.g., IEEE 802.11p or LTE-V2X) to set the frequency and time parameters. The first step is to set the amount of data symbols $F$ on each frame. Next, the total number of symbols is specified according to the standard. For the IEEE 802.11p, two training symbols are added at the beginning of each frame to obtain the initial channel estimation [11]. Therefore, each frame contains $K=F+2$ OFDM symbols. On the other hand, the number of symbols is fixed in the LTE-V2X specifications. There are 14 symbols in total, from which nine are used for data [14]. The next step is to establish the desired range of the energy per bit-to-noise spectral density ratio $(\mathrm{Eb} / \mathrm{No})$ to simulate data transmission over different noise levels. The parameters of the multicarrier scheme (number of data and total subcarriers, number of pilots) are then configured according to the specifications for the desired bandwidth. For instance, we can follow the parameters in Table 1 for a $10 \mathrm{MHz}$ bandwidth. Moreover, the specifications for DSRC and C-V2X communications differ in the type of pilots. In the case of IEEE 802.11p, it defines four subcarriers as pilots, whereas the LTE-V2X specifications consider four complete symbols. After defining those values, the parameters for the propagation scenario are established, and a matrix that represents the transmitted symbols (including data and pilots) is generated randomly for each noise level. Since the simulator is based on the Monte Carlo principle, $n$ realizations of the transmission process are necessary to obtain an average performance metric. For each iteration, a channel transfer function is calculated by calling the function GenerateChannel, which depends on the deterministic parameters described above. Subsequently, the data transmission is simulated following Equation (1). This simulator can be employed to evaluate different schemes, such as channel estimation. Therefore, we perform such transmission tasks and assess their performance over the generated channel. Finally, the BER is averaged for all iterations.

Table 4. Pseudocode for the simulation of V2X communications systems.

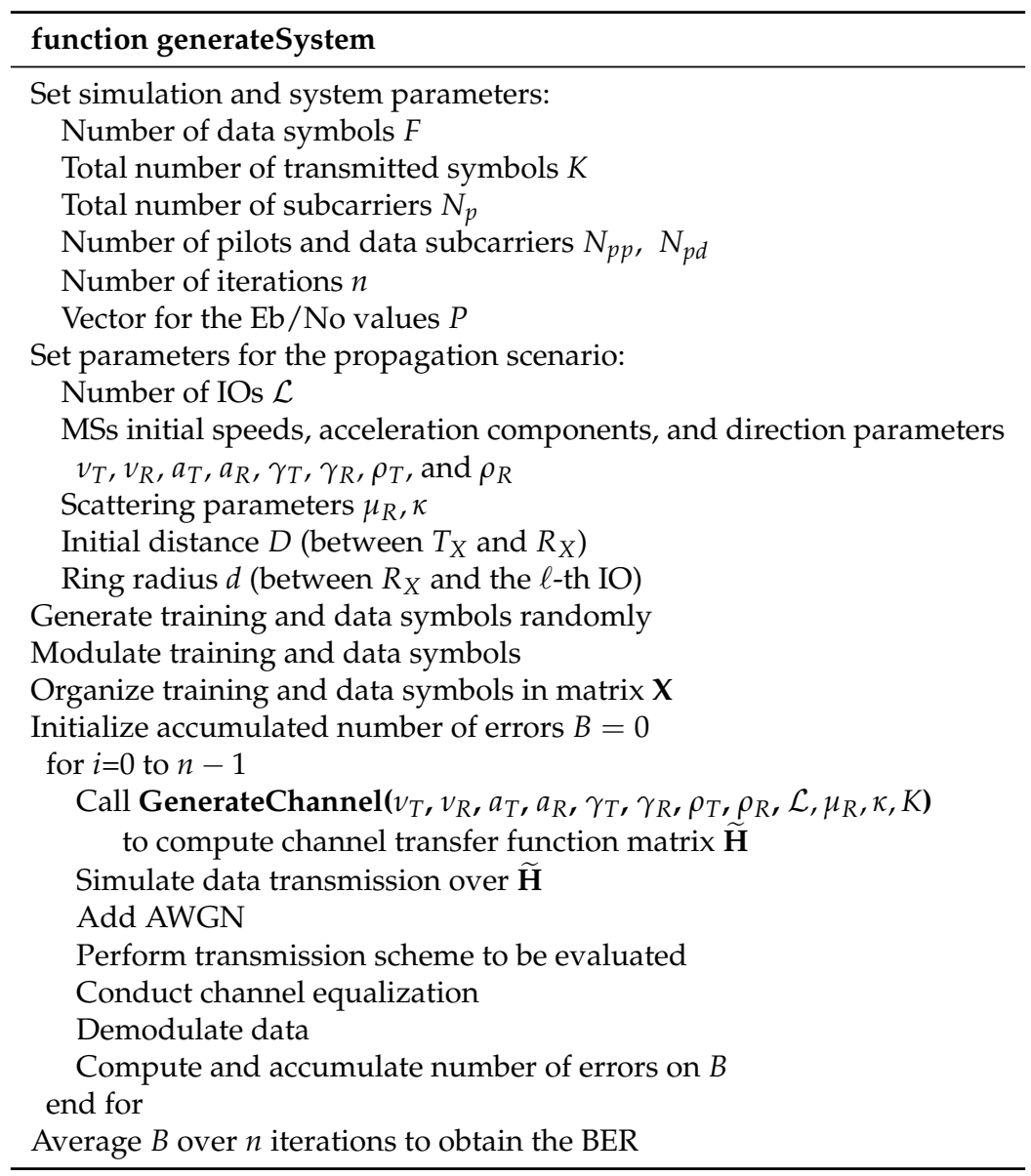




\section{Simulation Examples}

This section presents the performance analysis of a V2X communication system under different propagation scenarios employing binary phase-shift keying (BPSK) modulation. This framework can be adapted to any multicarrier system and modulation scheme, such as the ones specified by the IEEE 802.11p and 3GPP LTE-V2X standards. In particular, we used the proposed simulator to evaluate the BER performance of a DSRC system based on the IEEE $802.11 \mathrm{p}$ standard in scenarios where road safety applications might be employed. We evaluated the performance of two channel estimation techniques that are considered for IEEE 802.11p transceivers, namely the LS estimator and the STA technique, which are described in the following subsections.

\subsection{Channel Estimation Techniques for the IEEE 802.11p Standard}

Channel estimation and equalization techniques are widely used in wireless communications to recover the signal that propagated through the channel. In the IEEE 802.11p standard, channel estimation is performed using the LTSs $T_{1}$ and $T_{2}$ that are inserted in the preamble of each frame. For this standard, the signal model shown in (1) comprises both training and information data, in such a way that $\mathbf{Y}=\left[\mathbf{Y}^{\mathrm{e}} \mathbf{Y}^{\mathrm{d}}\right]$, $\mathbf{H}=\left[\mathbf{H}^{\mathrm{e}} \mathbf{H}^{\mathrm{d}}\right], \mathbf{X}=\left[\mathbf{X}^{\mathrm{e}} \mathbf{X}^{\mathrm{d}}\right]$, and $\mathbf{N}=\left[\mathbf{N}^{\mathrm{e}} \mathbf{N}^{\mathrm{d}}\right]$, where the superscripts $(\cdot)^{\mathrm{e}}$ and $(\cdot)^{\mathrm{d}}$ denote training data blocks and information data blocks, respectively; $\mathbf{Y}^{\mathrm{e}}, \mathbf{H}^{\mathrm{e}}, \mathbf{X}^{\mathrm{e}}$, and $\mathbf{N}^{\mathrm{e}}$ are $N_{p} \times 2$ complex-valued matrices; $\mathbf{Y}^{\mathrm{d}}, \mathbf{H}^{\mathrm{d}}, \mathbf{X}^{\mathrm{d}}$, and $\mathbf{N}^{\mathrm{d}}$ are $N_{p} \times F$ complex-valued matrices; and $F=K-2$ corresponds to the transmitted OFDM information data symbols on each frame. The OFDM training symbols are identical, so that $\mathbf{x}_{1}^{\mathrm{e}}=\mathbf{x}_{2}^{\mathrm{e}}$, where $\mathbf{x}_{k}^{\mathrm{e}} \in \mathbb{C}^{N_{p} \times 1}$ is the $k$-th column of the matrix $\mathbf{X}^{\mathrm{e}}$ for $k=1,2$.

\subsubsection{Least Squares Estimator}

The LS channel estimation over the $k$-th OFDM symbol and the $m$-th subcarrier is given by:

$$
\left\langle\widehat{\mathbf{H}}_{\llcorner s}^{\mathrm{d}}\right\rangle_{m, k}=\frac{\left\langle\mathbf{Y}^{\mathrm{e}}\right\rangle_{m, 1}+\left\langle\mathbf{Y}^{\mathrm{e}}\right\rangle_{m, 2}}{2 \times\left\langle\mathbf{X}^{\mathrm{e}}\right\rangle_{m, 1}}
$$

for $m=1,2, \ldots, N_{p}$ and $k=1,2, \ldots, F$, where $\widehat{\mathbf{H}}_{L s}^{\mathrm{d}}$ represents the estimated channel matrix. From (15), we can see that the LS estimator is used when we assume a time-invariant channel for the transmission of the whole frame, so that the estimated channel $\left\langle\widehat{\mathbf{H}}_{s s}^{\mathrm{d}}\right\rangle_{m, k}$ is the same for all $k$, i.e., for all OFDM information data symbols. After the channel estimation stage, the received OFDM symbols pass to an equalization process. Considering zero-forcing equalization, the equalized signal is given by:

$$
\left\langle\tilde{\mathbf{Y}}_{L s}^{\mathrm{d}}\right\rangle_{m, k}=\frac{\left\langle\mathbf{Y}^{\mathrm{d}}\right\rangle_{m, k}}{\left\langle\widehat{\mathbf{H}}_{L s}^{\mathrm{d}}\right\rangle_{m, k}}
$$

for $m=1,2, \ldots, N_{p}$ and $k=1,2, \ldots, F$, where $\tilde{\mathbf{Y}}_{L S}^{\mathrm{d}}$ is the equalized data matrix.

\subsubsection{Spectral Temporal Averaging Technique}

The STA technique presented in [32] takes as the initial point the LS estimator in (15). This estimation scheme is intended for time-varying channels, as it tracks the channel variations according to:

$$
\left\langle\tilde{\mathbf{Y}}_{\text {sTA }}^{\mathrm{d}}\right\rangle_{m, k}=\frac{\left\langle\mathbf{Y}^{\mathrm{d}}\right\rangle_{m, k}}{\left\langle\widehat{\mathbf{H}}_{s T A}^{\mathrm{d}}\right\rangle_{m, k-1}}
$$

for $m=1,2, \ldots, N_{p}$ and $k=2,3, \ldots, F$, where $\widehat{\mathbf{H}}_{S T A}^{\mathrm{d}}$ and $\tilde{\mathbf{Y}}_{\text {STA }}^{\mathrm{d}}$ are the estimated channel and the equalized data matrix, respectively, when the STA technique is employed. In the STA technique, the $k$-th equalized symbol represented by $\left\langle\tilde{\mathbf{Y}}_{\text {sTA }}^{\mathrm{d}}\right\rangle_{m, k}$ depends on the estimate $\left\langle\widehat{\mathbf{H}}_{\text {STA }}^{\mathrm{d}}\right\rangle_{m, k-1}$ computed from the previous data symbol. The first estimate $\left\langle\widehat{\mathbf{H}}_{\text {sTA }}^{\mathrm{d}}\right\rangle_{m, 1}=\left\langle\widehat{\mathbf{H}}_{L S}^{\mathrm{d}}\right\rangle_{m, 1}$ corresponds to that obtained with the LS scheme from (15). 
Then, the equalized symbol $\left\langle\tilde{\mathbf{Y}}_{\text {STA }}^{\mathrm{d}}\right\rangle_{m, k}$ shown in (17) is demodulated in order to obtain an estimate $\left\langle\widehat{\mathbf{X}}^{\mathrm{d}}\right\rangle_{m, k}$ of the transmitted OFDM data symbols, where $\widehat{X}^{\mathrm{d}}$ represents the estimation of the transmitted data matrix. Such estimates are used to obtain dynamic channel estimations based on:

$$
\left\langle\widehat{\mathbf{H}}_{A c T}^{\mathrm{d}}\right\rangle_{m, k}=\frac{\left\langle\mathbf{Y}^{\mathrm{d}}\right\rangle_{m, k}}{\left\langle\widehat{\boldsymbol{X}}^{\mathrm{d}}\right\rangle_{m, k}}
$$

for $m=1,2, \ldots, N_{p}$ and $k=2,3, \ldots, F$ where $\widehat{\mathbf{H}}_{A C T}^{\mathrm{d}}$ denotes an estimate of the channel matrix.

The estimates $\left\langle\widehat{\mathbf{H}}_{A c T}^{\mathrm{d}}\right\rangle_{m, k}$ given in (18) are affected by AWGN. For this reason, an averaging operation is computed for each subcarrier as follows:

$$
\left\langle\overline{\mathbf{H}}^{\mathrm{d}}\right\rangle_{m, k}=\frac{1}{2 \beta+1} \sum_{n=-\beta}^{n=+\beta}\left\langle\widehat{\mathbf{H}}_{A c T}^{\mathrm{d}}\right\rangle_{m, k+n}
$$

for $m=1,2, \ldots, N_{p}$ and $k=2,3, \ldots, F$, where $\overline{\mathbf{H}}^{\mathrm{d}}$ is a matrix that contains all the averages in the frequency domain and $\beta$ is an integer variable that controls the number of subcarriers in the averaging process. In general, when we have a frequency selective channel, the value of $\beta$ must be small. On the other hand, for non-selective channels $\beta$ must be higher [32]. Then, the estimated channel is given by:

$$
\left\langle\widehat{\mathbf{H}}_{s T A}^{\mathrm{d}}\right\rangle_{m, k}=\left(1-\frac{1}{\alpha}\right)\left\langle\widehat{\mathbf{H}}_{s T A}^{\mathrm{d}}\right\rangle_{m, k-1}+\frac{1}{\alpha}\left\langle\overline{\mathbf{H}}^{\mathrm{d}}\right\rangle_{m, k}
$$

for $m=1,2, \ldots, N_{p}$ and $k=2,3, \ldots, F$, where $\alpha$ is an updating and weighting factor restricted to $\alpha>1$ and $(1-1 / \alpha)>0$. A large value of $\alpha$ is used in slow-fading channels, while a small value is preferred for fast-fading channels [32]. Finally, the channel estimation shown in Equation (20) is used in (17) to equalize the next OFDM data symbol and repeat the whole process until the end of the frame.

\subsection{Simulation Setup}

For the simulation experiments, we considered the geometrical one-ring scattering model with a ring of IOs surrounding the $R_{X}$ under isotropic $(\kappa=0)$ and non-isotropic $(\kappa>0)$ scattering conditions. The propagation scenarios were defined in such a way that they resemble situations where road safety applications might have a fundamental role. The evaluated scenarios are: (i) an intersection where two vehicles are approaching each other; (ii) an overtaking maneuver where a vehicle changes its trajectory to pass the one in the front; and (iii) a highway situation with two vehicles moving on opposite lanes. We assumed a set of general parameters to compare the performance in the different propagation scenarios. The number of IOs surrounding the receiver was $\mathcal{L}=20$, the initial distance between $T_{X}$ and $R_{X}: D=500 \mathrm{~m}$, and the distance of the ring of IOs surrounding $R_{X}: d=30 \mathrm{~m}$. For the cases that include acceleration components $\left(a_{T}\right.$ and $\left.a_{R}\right)$, we considered a magnitude of $20 \mathrm{~m} / \mathrm{s}^{2}$. We acknowledge that these acceleration terms are extremely high for an urban environment, but they help us to illustrate that despite their excessive magnitudes, the performance differences from a constant velocity scenario are almost imperceptible by the small transmission times. The particular parameters that define the motion of the MSs for each scenario are described below.

- Scenario I: Intersection. The MSs move in such a way that they approach each other with angles $\gamma_{T}=60^{\circ}$ and $\gamma_{R}=120^{\circ}$, and both $T_{X}$ and $R_{X}$ acceleration components point in the same direction of their corresponding initial speeds (i.e., $\gamma_{T}=\rho_{T}$ and $\gamma_{R}=\rho_{R}$ ). The motion of the MSs resembles a safety scenario where vehicles circulate on two streets that intersect at one point. This scenario is depicted in Figure 5.

- Scenario II: Overtaking maneuver. A vehicle accelerates and changes its direction of motion aiming to overtake the one in front of it. For this scenario, we assume that both vehicles start 
moving in the same direction $\gamma_{T}=\gamma_{R}=0^{\circ}$, but the transmitting station has an acceleration component with angle $\gamma_{R}=15^{\circ}$, which modifies its trajectory over time. In the case of the receiving vehicle, this one has no acceleration component, i.e., $\mathbf{a}_{R}=\mathbf{0}$. Figure 6 illustrates this scenario where the two vehicles start on the same lane.

- Scenario III: Opposite lanes. Two vehicles are approaching each other on opposite parallel lanes of a highway environment. This scenario is shown in Figure 7, where we consider the angles $\gamma_{T}=\rho_{T}=-10^{\circ}$ and $\gamma_{R}=\rho_{R}=170^{\circ}$ to describe the motion of the vehicles in opposite directions over parallel lanes.

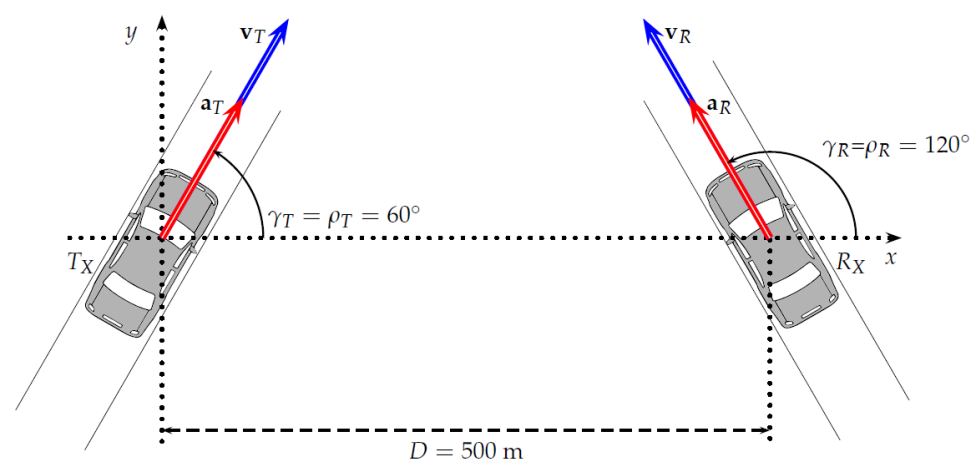

Figure 5. Scenario I: Propagation scenario reproducing a safety situation, where two vehicles are approaching an intersection on the road.

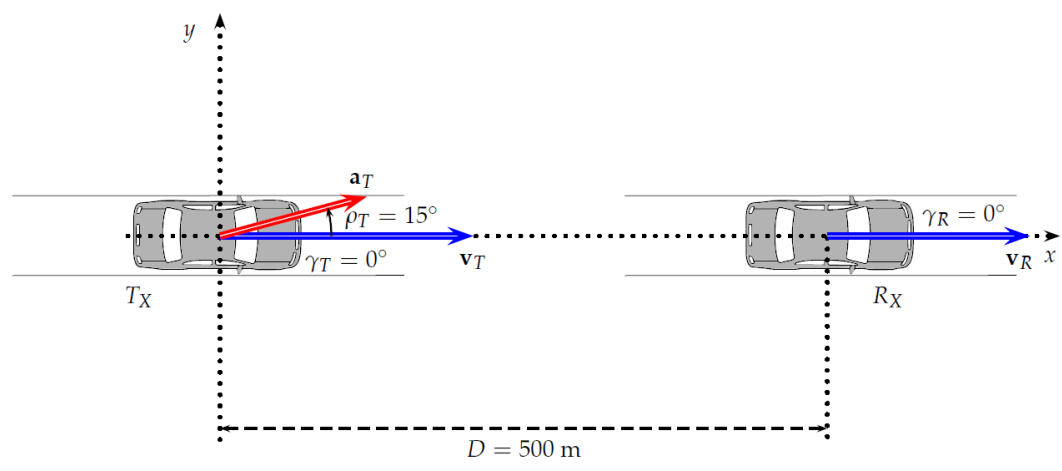

Figure 6. Scenario II: Propagation scenario that recreates an overtaking maneuver, where a vehicle changes its lane before passing another in front.

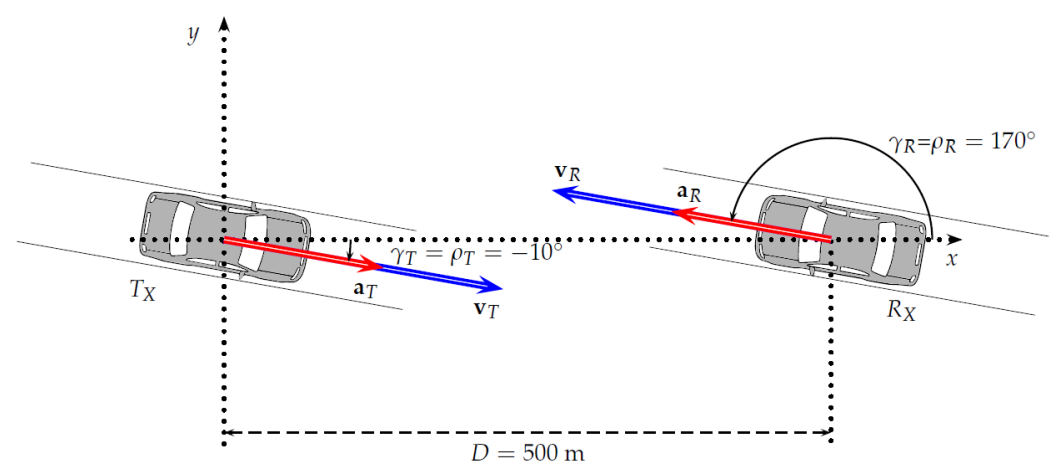

Figure 7. Scenario III: Propagation scenario resembling a highway situation, where two vehicles are approaching on opposite lanes. 


\subsection{Results of Changes of the Initial Speed}

First, we evaluated the BER performance for a transmission of $F=64$ data OFDM symbols in each frame at different relative speeds between the MSs by varying both the initial speed and acceleration components. For this simulation, a propagation environment with isotropic scattering was considered $(\kappa=0)$, that is a scenario that resembles an urban environment with IOs that uniformly surround $R_{X}$. The BER performance curves for Scenario I are shown in Figure 8, for both the LS estimator and the STA technique. In the case of the LS estimator, the performance decreased when the initial speeds of the MSs were increased from $v_{T}=v_{R}=20 \mathrm{~km} / \mathrm{h}$ (relative speed of $40 \mathrm{~km} / \mathrm{h}$ ) to $v_{T}=v_{R}=100 \mathrm{~km} / \mathrm{h}$ (relative speed of $200 \mathrm{~km} / \mathrm{h}$ ) regardless of the propagation scenario. This condition occurred since the Doppler frequency shifts increases as the relative speed also increases. Therefore, the channel presents more variations, and the LS estimation becomes less accurate.

In fact, the BER performance of the LS estimator is independent of the channel non-stationarities and MSs acceleration components since the channel estimation is carried out with the two initial training symbols on each frame. Consequently, the time variations in a data frame caused by the non-stationarities and acceleration terms could be negligible for the LS estimator. This result is illustrated in Figure 8, which is consistent with [56] for WSSUS channels and with [57] for non-WSSUS channels with constant speed. Concerning the STA technique, the BER performance was poor compared to the LS estimator. Such performance was almost equal to the case where there is no channel estimation at all since the statistics of the non-WSSUS process change as the data transmission proceeds, and the non-stationarities directly impact the weighted average that was carried out in the frequency domain $[57,58]$. In this particular case, the channel effect on the system performance due to the acceleration of the MSs was minimal and masked by its inherent non-stationarities [59].

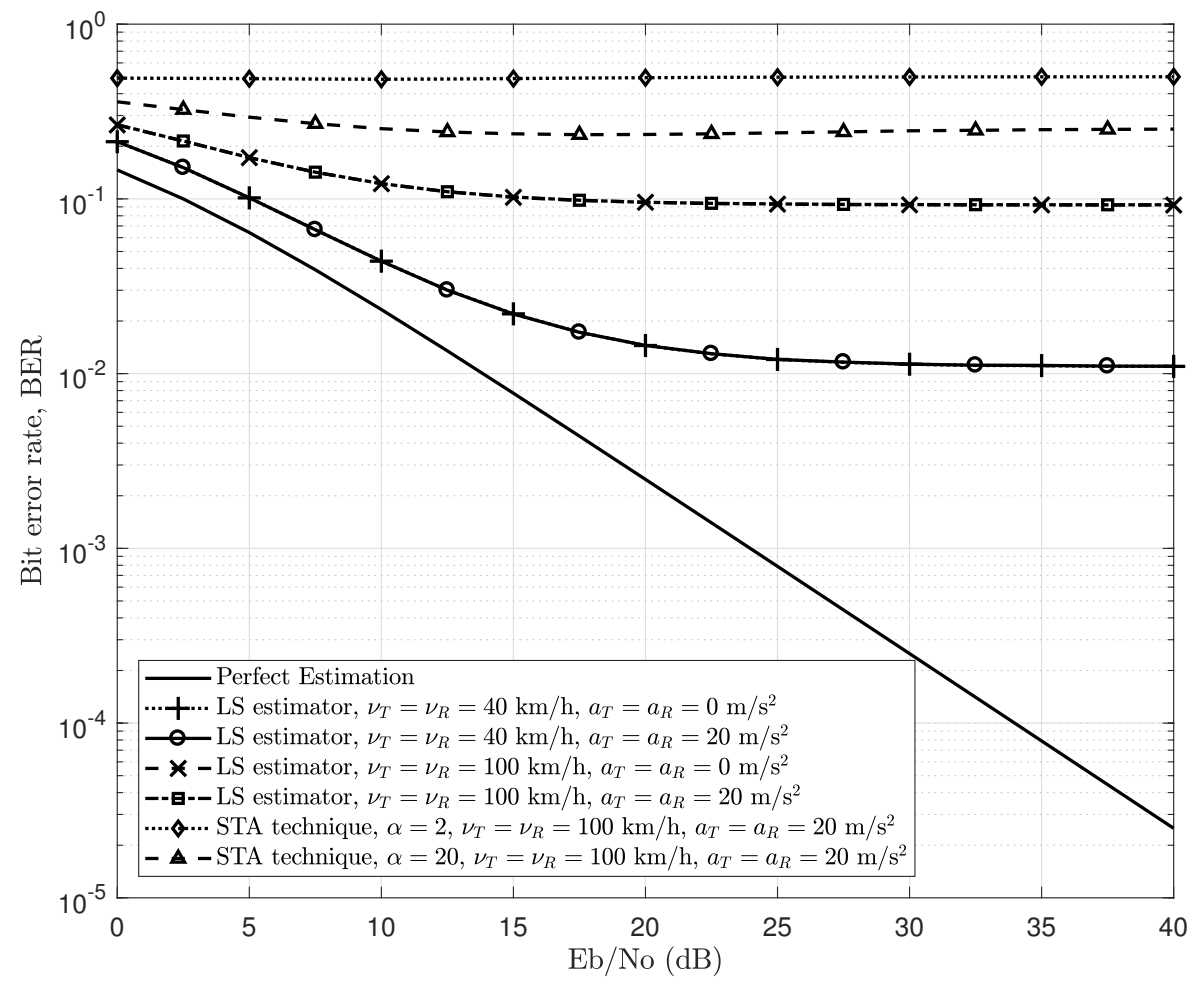

Figure 8. System performance by considering different initial speeds and acceleration components for Scenario I with a constant frame length of $F=64$.

In addition to the results presented in Figure 8, Table 5 shows the Eb/No levels that attained 10\% and $1 \%$ BER for the low mobility $(20 \mathrm{~km} / \mathrm{h}$ and $40 \mathrm{~km} / \mathrm{h})$ and high mobility $(80 \mathrm{~km} / \mathrm{h}$ and $100 \mathrm{~km} / \mathrm{h})$ 
conditions in the three propagation scenarios described above. The legend NA denotes that the BER level was not achieved by using the corresponding estimation scheme. In this table, we can observe that the performance of the LS estimator in low mobility conditions was similar for the three scenarios, reaching 10\% BER with comparable Eb/No levels. In such conditions, an Eb/No regime greater than $5.5 \mathrm{~dB}$ guarantees a BER lower than $10 \%$ for all the scenarios. On the other hand, for high mobility conditions, we observed that $\mathrm{Eb} / \mathrm{No}$ levels greater than $10 \mathrm{~dB}$ were required to attain a $10 \% \mathrm{BER}$. As observed in Figure 8, the BER performance of this estimator was greater than $0.1 \%$ even at high $\mathrm{Eb} /$ No regimes for initial speeds of $100 \mathrm{~km} / \mathrm{h}$. Furthermore, the results showed that the performance of the LS estimation in Scenario I was the least affected by the high mobility conditions, reaching BER levels lower than $10 \%$ with an $\mathrm{Eb} /$ No greater than $16.51 \mathrm{~dB}$ when the initial speeds were $100 \mathrm{~km} / \mathrm{h}$. For the STA technique, its BER performance always remained greater than $10 \%$ in these experiments regardless of the propagation scenario although using a large value of $\alpha$.

Table 5. Required energy per bit-to-noise spectral density ratio (Eb/No) levels to attain $10 \%$ and $1 \%$ BER over Scenarios I, II, and III at different initial speeds by considering acceleration components of $a_{T}=a_{R}=20 \mathrm{~m} / \mathrm{s}^{2}$ and a constant frame length of $F=64$ (NA-not achieved).

\begin{tabular}{|c|c|c|c|c|c|c|c|c|c|c|c|c|c|}
\hline & & \multicolumn{4}{|c|}{ Scenario I } & \multicolumn{4}{|c|}{ Scenario II } & \multicolumn{4}{|c|}{ Scenario III } \\
\hline \multicolumn{2}{|c|}{$\begin{array}{l}\text { Initial MS speed } \\
v_{T}=v_{R}(\mathrm{~km} / \mathrm{h})\end{array}$} & 20 & 40 & 80 & 100 & 20 & 40 & 80 & 100 & 20 & 40 & 80 & 100 \\
\hline Estimator & BER & \multicolumn{12}{|c|}{ Required Eb/No (dB) } \\
\hline LS & $10^{-1}$ & 4.73 & 5.12 & 8.69 & 16.51 & 4.67 & 5.37 & 13.54 & NA & 4.81 & 5.29 & 12.88 & NA \\
\hline STA $(\alpha=2)$ & $10^{-1}$ & NA & NA & NA & NA & NA & NA & NA & NA & NA & NA & NA & NA \\
\hline $\operatorname{STA}(\alpha=20)$ & $10^{-1}$ & $\overline{\mathrm{NA}}$ & $\overline{\mathrm{NA}}$ & $\mathrm{NA}$ & $\overline{\mathrm{NA}}$ & NA & $\mathrm{NA}$ & $\overline{\mathrm{NA}}$ & $\mathrm{NA}$ & $\mathrm{NA}$ & $\mathrm{NA}$ & $\mathrm{NA}$ & $\mathrm{NA}$ \\
\hline LS & $10^{-2}$ & 16.99 & NA & NA & NA & 16.94 & NA & NA & $\mathrm{NA}$ & 16.91 & NA & NA & $\mathrm{NA}$ \\
\hline STA $(\alpha=2)$ & $10^{-2}$ & NA & NA & NA & NA & NA & NA & NA & NA & NA & NA & NA & NA \\
\hline STA $(\alpha=20)$ & $10^{-2}$ & NA & NA & NA & NA & NA & NA & NA & NA & NA & NA & NA & NA \\
\hline
\end{tabular}

\subsection{Results of Changes of the Frame Length}

For these simulation experiments, the BER performance was evaluated in low mobility conditions transmitting different numbers of OFDM data symbols by considering initial speeds of $v_{T}=v_{R}=40 \mathrm{~km} / \mathrm{h}$, acceleration magnitudes of $a_{T}=a_{R}=20 \mathrm{~m} / \mathrm{s}^{2}$, and a scenario with isotropic scattering $(\kappa=0)$. Figure 9 shows that the BER performance of the LS estimation in Scenario I decreased as the size of the frame increased. This drop in performance is due to the channel variations between the transmitted OFDM data symbols, which causes the initial LS estimation to gradually lose its validity until it becomes practically obsolete for very large frames. In this case, the worst performance for the LS estimator occurred after the frame length exceeded 128 OFDM data symbols. Regarding the STA technique, the results showed that as the updating factor increased (e.g., from $\alpha=2$ to $\alpha=20$ ), the performance of the STA technique improved, as shown in Figure 9. In such a case, as the parameter $\alpha$ in (20) increased, the estimated channel tends to be similar to the LS estimation. Furthermore, the performance of the STA technique was benefited in low mobility scenarios with small frame lengths, as observed for $F=32$. Finally, by increasing the frame length and/or decreasing the updating factor $\alpha$, the performance of the STA technique approached the case where there was no channel estimation because the weighted average in (20) became imprecise in non-WSSUS channels, whose statistics changed before the transmission of a data frame ended [58].

Table 6 presents the required Eb/No levels to achieve $10 \%$ and $1 \%$ BER by employing the LS and STA techniques in low mobility conditions, assuming frame lengths ranging from 16 to 128 OFDM symbols in the three propagation scenarios described previously. This table shows that as the frame length increased, the performance of both the LS estimator and STA technique with $\alpha=20$ decreased. As observed in Table 5 for changes in the initial speed, the BER performance of the STA technique with $\alpha=2$ always remained greater than $10 \%$. For frames of 64 OFDM symbols or less, the performance of 
the LS and STA (with $\alpha=20$ ) techniques was similar in the three considered scenarios approaching the $10 \%$ BER with comparable Eb/No levels. In particular, in the case of short frames with 32 symbols or less, an Eb/No level greater than $4.61 \mathrm{~dB}$ and $7.15 \mathrm{~dB}$ for the LS estimator and STA technique (with $\alpha=20$ ), respectively, guaranteed a BER lower than $10 \%$ for all the propagation scenarios. Furthermore, for these short frame lengths, the performance of the LS estimator and STA technique with $\alpha=20$ reached $1 \%$ for $\mathrm{Eb} / \mathrm{No}$ regimes greater than $17.06 \mathrm{~dB}$ and $39.99 \mathrm{~dB}$, respectively. Finally, Eb/No levels greater than $5.41 \mathrm{~dB}$ and $10.7 \mathrm{~dB}$ reached BER performances lower than 10\% employing the LS estimator for frame lengths of 64 and 128, respectively. For the STA technique, considering frames with more than 64 OFDM symbols, the BER performance always remained greater than $1 \%$ when using $\alpha=20$. As observed, the STA estimator performed well assuming short data frames and low mobility conditions. However, this technique demanded larger $\mathrm{Eb} / \mathrm{No}$ values than the LS estimator.

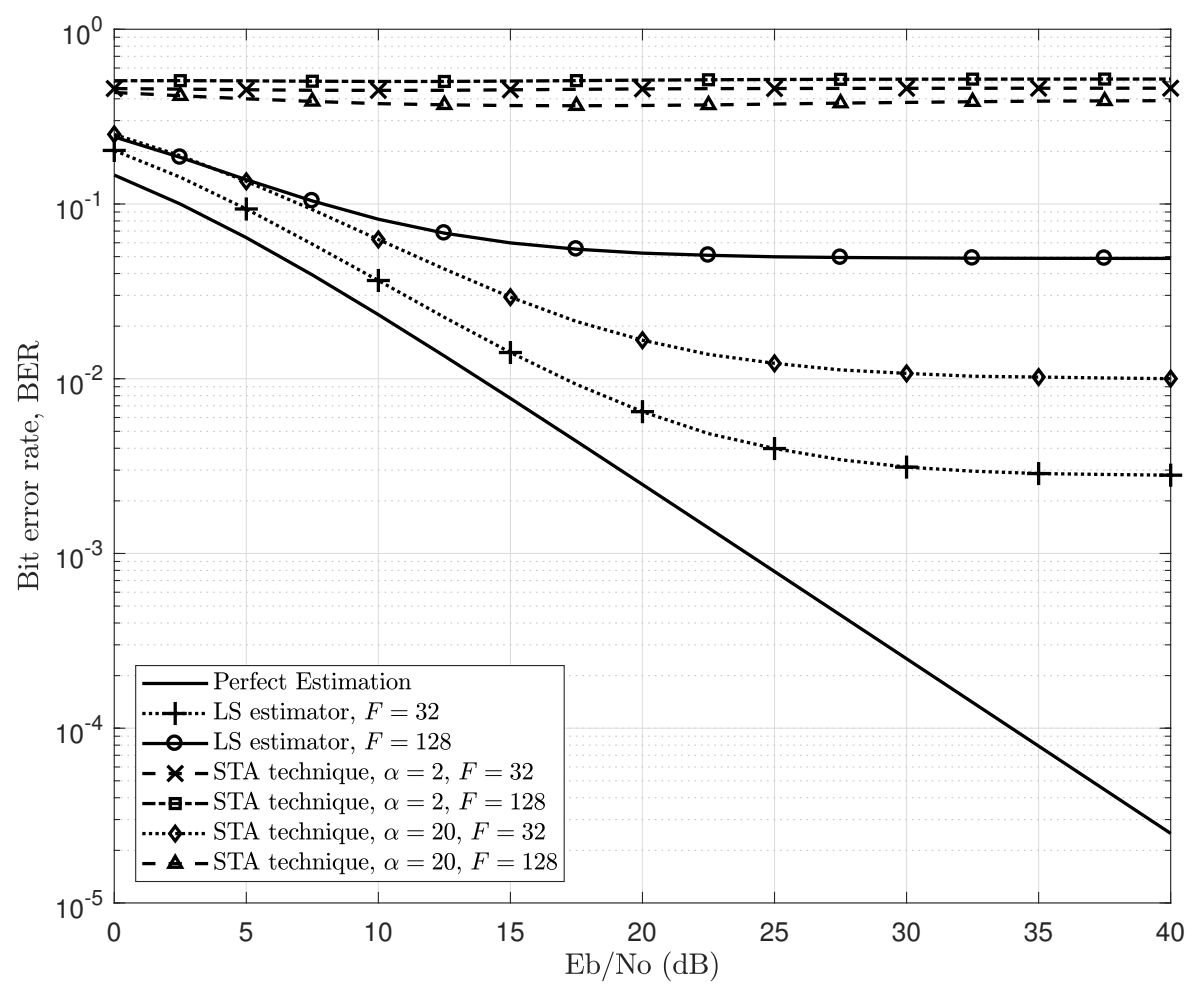

Figure 9. System performance by considering different frame lengths with initial speeds of $v_{T}=v_{R}=40 \mathrm{~km} / \mathrm{h}$ and acceleration components of $a_{T}=a_{R}=20 \mathrm{~m} / \mathrm{s}^{2}$ for Scenario I.

Table 6. Required Eb/No levels to attain 10\% and 1\% BER over Scenarios I, II, and III for different frame lengths by considering initial speeds of $v_{T}=v_{R}=40 \mathrm{~km} / \mathrm{h}$ and acceleration components of $a_{T}=a_{R}=20 \mathrm{~m} / \mathrm{s}^{2}$ (NA-not achieved).

\begin{tabular}{cccccccccccccc}
\hline & \multicolumn{3}{c}{ Scenario I } & \multicolumn{3}{c}{ Scenario II } \\
\hline $\begin{array}{c}\text { Frame length } \\
F\end{array}$ & 16 & 32 & 64 & 128 & 16 & 32 & 64 & 128 & 16 & 32 & 64 & 128 \\
\hline Estimator & BER & & & \multicolumn{1}{c}{ Required $\mathrm{Eb} / \mathrm{No}(\mathrm{dB})$} \\
\hline LS & $10^{-1}$ & 4.00 & 4.61 & 5.08 & 7.92 & 3.98 & 4.52 & 5.37 & 10.70 & 3.87 & 4.55 & 5.41 & 10.31 \\
\hline STA $(\alpha=2)$ & $10^{-1}$ & NA & NA & NA & NA & NA & NA & NA & NA & NA & NA & NA & NA \\
\hline STA $(\alpha=20)$ & $10^{-1}$ & 4.48 & 7.05 & NA & NA & 4.50 & 7.15 & NA & NA & 4.43 & 6.98 & NA & NA \\
\hline LS & $10^{-2}$ & 15.37 & 17.06 & NA & NA & 15.34 & 16.63 & NA & NA & 15.18 & 16.79 & NA & NA \\
\hline STA $(\alpha=2)$ & $10^{-2}$ & NA & NA & NA & NA & NA & NA & NA & NA & NA & NA & NA & NA \\
\hline STA $(\alpha=20)$ & $10^{-2}$ & 16.10 & 34.38 & NA & NA & 15.66 & 39.99 & NA & NA & 15.79 & 32.36 & NA & NA \\
\hline
\end{tabular}




\subsection{Results over Non-Isotropic Scattering}

The following experiment was carried out with the objective of analyzing scenarios where the IOs that surrounded the $R_{X}$ were distributed in a particular region. This condition emulates a scenario where there is a large amount of free space and the IOs are concentrated in one given direction, e.g., rural environments. In this simulation, high mobility conditions with initial speeds of $v_{T}=v_{R}=100 \mathrm{~km} / \mathrm{h}$, acceleration magnitudes of $a_{T}=a_{R}=20 \mathrm{~m} / \mathrm{s}^{2}$, and a fixed frame length of $F=32$ were considered to transmit data over highly non-isotropic scattering scenarios with $\kappa=20$ and three different means $\mu_{R}=0, \pi / 2$, and $3 \pi / 4$. We compared the results with the isotropic scenario using $\kappa=0$. Figure 10 shows the BER performance curves for this experiment according to Scenario I (see Figure 5). The plot shows that the performance of the LS estimator improved remarkably in non-isotropic conditions, i.e., when the IOs were concentrated in a region. In this scenario, the maximum propagation delay decreases as the IOs concentrate in a region that is closer to the transmitting vehicle, so the frequency selectivity of the channel is reduced [42]. Furthermore, the performance of the LS estimator depends on the mean $\mu_{R}$ of the AOAs and also on the directions of motion $\left(\gamma_{T}\right.$ and $\left.\gamma_{R}\right)$ of the MSs. For example, taking as a reference the propagation scenario shown in Figure 5, assuming $\kappa=20, \mu_{R}=3 \pi / 4, \gamma_{T}=\rho_{T}=60^{\circ}$, and $\gamma_{R}=\rho_{R}=120^{\circ}$, the IOs were concentrated in a region that was closer to $T_{X}$, so that the maximum propagation delay was smaller, which benefited the performance of the LS estimator. Similarly, by considering $\mu_{R}=\pi / 2$ or $\mu_{R}=0$, which meant that the IOs were further away from $T_{X}$, the maximum propagation delay was larger resulting a more frequency-selective channel, and as a consequence, a poorer performance of the LS estimator. Finally, the LS estimator performance was almost insignificantly affected by the magnitude and direction of the MSs acceleration (i.e., $a_{T}, a_{R}, \rho_{T}$, and $\rho_{R}$ ) since the estimation was carried out by the two initial OFDM training symbols on the frame. These results are consistent with [57] for non-WSSUS channels, where there are no acceleration components. In the case of the STA technique, the main drop in its performance was caused by the channel non-stationarities, regardless of the initial speed or acceleration components of the MSs. This was due to the dynamic channel estimation in (18), which becomes obsolete rapidly even between two consecutive OFDM symbols. Therefore, although considering the data transmission over highly non-isotropic scattering scenarios, the performance of the STA technique with small values of $\alpha$ always approaches the case where there is no channel estimation, as observed in Figure 10.

Table 7 shows the required Eb/No levels to attain 10\%, 1\%, and $0.1 \%$ BER over the described propagation scenarios for isotropic and non-isotropic scattering conditions. We considered initial speeds of $v_{T}=v_{R}=100 \mathrm{~km} / \mathrm{h}$ and acceleration components of $a_{T}=a_{R}=20 \mathrm{~m} / \mathrm{s}^{2}$, with a constant frame length of $F=32$ OFDM symbols. The results showed that the BER performance of both LS and STA (with $\alpha=20$ ) techniques depended on the placement of the IOs. This behavior occurred since the propagation delays depends on the AOAs, which are determined by the location of the IOs that surround the receiving vehicle. The BER performance decreases when the maximum propagation delay increases, which occurrs when the IOs were located further from the transmitting vehicle. Therefore, the initial positions and motion profiles of the MSs also impacted the BER performance in non-isotropic conditions. The BER performance of both estimation techniques in isotropic conditions was always lower than for the non-isotropic scenarios. Such performance was always greater than $1 \%$ in the considered simulation conditions. In the case of the LS estimator, its performance attained a $10 \% \mathrm{BER}$ with similar Eb/No levels. However, depending on the mean $\mathrm{AOA} \mu_{R}$, the $\mathrm{Eb} / \mathrm{No}$ requirement could increase significantly. For instance, in Scenario II, a 10\% BER performance was yielded with 4.43 and $8.14 \mathrm{~dB}$, when the mean AOAs were $3 \pi / 4$ and 0 , respectively. With respect to the STA scheme with $\alpha=20$, its performance was also affected by the mean AOA that defined the region where the IOs concentrated. This technique required higher $\mathrm{Eb} / \mathrm{No}$ levels than observed for the LS estimator. In Scenario III, a 10\% BER could be reached employing 7.03 and $19.03 \mathrm{~dB}$, when the IOs were located around 0 and $3 \pi / 4$, respectively, which was a significant increase in the $\mathrm{Eb} / \mathrm{No}$ 
requirement. In addition, the performance of the LS estimator could reach a $0.1 \%$ BER for high Eb/No regimes in some of the non-isotropic cases.

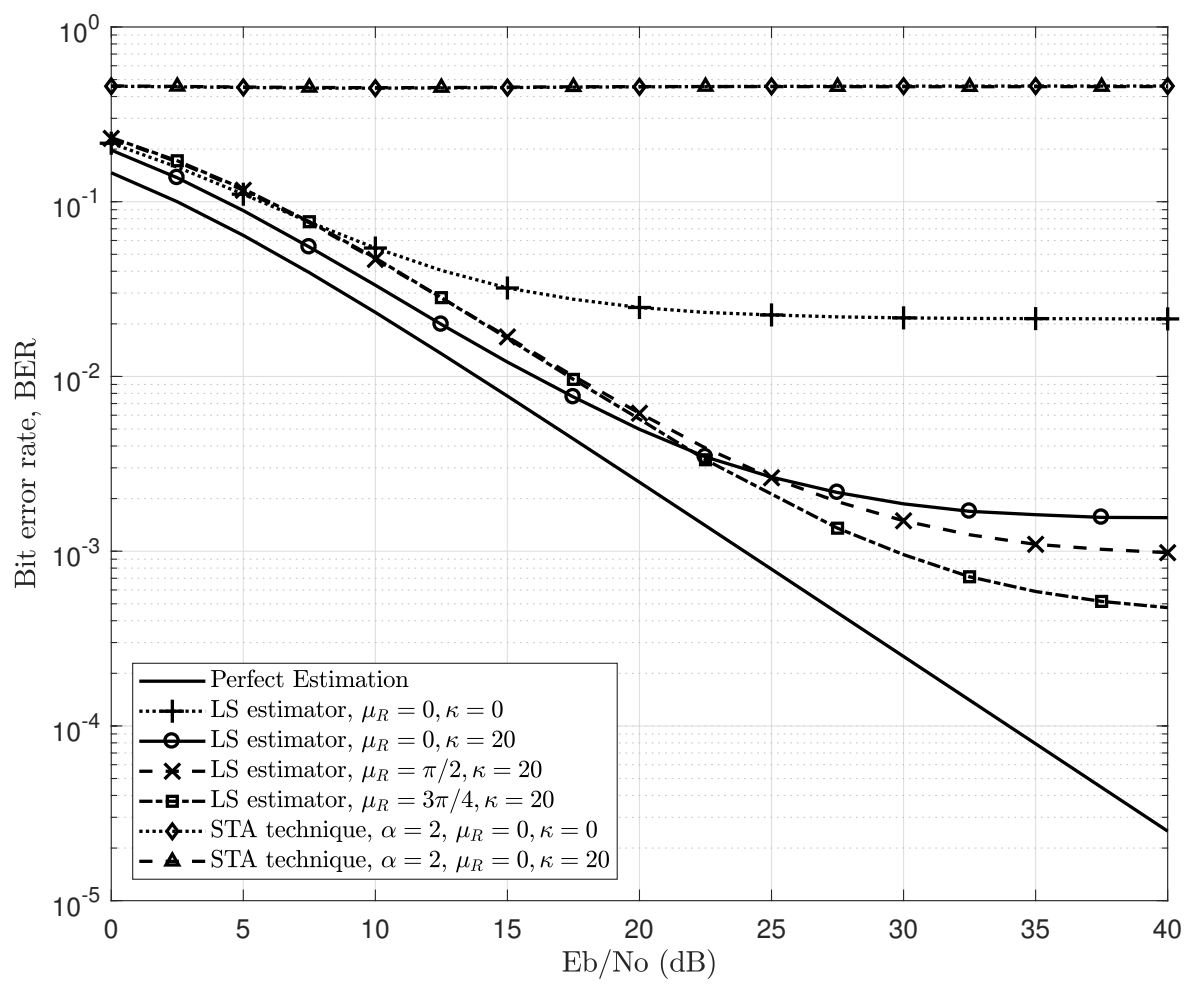

Figure 10. System performance over isotropic and non-isotropic scattering conditions with initial speeds of $v_{T}=v_{R}=100 \mathrm{~km} / \mathrm{h}$ and acceleration components of $a_{T}=a_{R}=20 \mathrm{~m} / \mathrm{s}^{2}$ for Scenario I, by assuming a constant frame length of $F=32$.

Table 7. Required Eb/No levels to attain $10 \%, 1 \%$, and $0.1 \%$ BER over Scenarios I, II, and III for isotropic and non-isotropic scattering conditions by considering initial speeds of $v_{T}=v_{R}=100 \mathrm{~km} / \mathrm{h}$ and acceleration components of $a_{T}=a_{R}=20 \mathrm{~m} / \mathrm{s}^{2}$, with a constant frame length of $F=32$ (NA-not achieved).

\begin{tabular}{cccccccccccccc}
\hline & \multicolumn{3}{c}{ Scenario I } & \multicolumn{3}{c}{ Scenario II } & \multicolumn{3}{c}{ Scenario III } \\
\hline $\begin{array}{c}\text { Mean AOA } \\
\mu_{R}\end{array}$ & $\begin{array}{c}0 \\
(\kappa=0)\end{array}$ & 0 & $\frac{\pi}{2}$ & $\frac{3 \pi}{4}$ & $\begin{array}{c}0 \\
(\kappa=0)\end{array}$ & 0 & $\frac{\pi}{2}$ & $\frac{3 \pi}{4}$ & $(\kappa=0)$ & 0 & $\frac{\pi}{2}$ & $\frac{3 \pi}{4}$ \\
\hline Estimator & BER & & & \multicolumn{1}{c}{ Required Eb/No (dB) } \\
\hline LS & $10^{-1}$ & 5.67 & 4.32 & 5.87 & 5.88 & 6.11 & 8.14 & 5.12 & 4.43 & 6.06 & 4.27 & 5.44 & 7.16 \\
\hline STA $(\alpha=2)$ & $10^{-1}$ & NA & NA & NA & NA & NA & NA & NA & NA & NA & NA & NA & NA \\
\hline STA $(\alpha=20)$ & $10^{-1}$ & 9.18 & 6.98 & 11.86 & 12.02 & 10.78 & NA & 9.00 & 6.94 & 10.85 & 7.03 & 9.67 & 19.03 \\
\hline LS & $10^{-2}$ & NA & 16.03 & 17.53 & 17.33 & NA & 20.14 & 17.35 & 15.88 & NA & 15.11 & 17.76 & 19.89 \\
\hline STA $(\alpha=2)$ & $10^{-2}$ & NA & NA & NA & NA & NA & NA & NA & NA & NA & NA & NA & NA \\
\hline STA $(\alpha=20)$ & $10^{-2}$ & NA & 22.09 & NA & NA & NA & NA & NA & 21.18 & NA & 18.98 & NA & NA \\
\hline LS & $10^{-3}$ & NA & NA & 38.90 & 29.67 & NA & 31.93 & NA & 39.75 & NA & 24.94 & NA & NA \\
\hline STA $(\alpha=2)$ & $10^{-3}$ & NA & NA & NA & NA & NA & NA & NA & NA & NA & NA & NA & NA \\
\hline STA $(\alpha=20)$ & $10^{-3}$ & NA & NA & NA & NA & NA & NA & NA & NA & NA & 32.82 & NA & NA \\
\hline
\end{tabular}

\subsection{Results of the STA Modification Proposal}

Since the reference model in (2) was developed for non-WSSUS channels, its second order statistics depend on both time and frequency, as demonstrated in [42]. Consequently, the averages made with the STA technique in the frequency domain are not entirely accurate in this type of channel, as can be 
seen in the simulation experiments presented earlier in this work. Therefore, we propose a modification to the STA technique that consists of eliminating the frequency averaging process for each subcarrier shown in (19), so that the new channel estimation is given by:

$$
\left\langle\widehat{\mathbf{H}}_{\text {STA }}^{\mathrm{d}}\right\rangle_{m, k}=\left(1-\frac{1}{\alpha}\right)\left\langle\widehat{\mathbf{H}}_{s T A}^{\mathrm{d}}\right\rangle_{m, k-1}+\frac{1}{\alpha}\left\langle\widehat{\mathbf{H}}_{A C T}^{\mathrm{d}}\right\rangle_{m, k}
$$

where $\left\langle\widehat{\mathbf{H}}_{A C T}^{\mathrm{d}}\right\rangle_{m, k}$ is the dynamic channel estimation shown in (18).

The BER performance curves corresponding to Scenario I by using the proposed estimation in (21) are shown in Figure 11. We assumed isotropic scattering $(\kappa=0)$ in a low mobility scenario at initial speeds of $v_{T}=v_{R}=40 \mathrm{~km} / \mathrm{h}$, acceleration magnitudes of $a_{T}=a_{R}=20 \mathrm{~m} / \mathrm{s}^{2}$, and frame lengths of $F=32$ and 64 , and 128 OFDM symbols. We can observe that the performance of the STA technique obtained with (21) improved significantly in comparison to the case where the frequency averaging process was included. Even if the MSs were accelerating, the frame length was increased or a small value of $\alpha$ was used for the estimation process, and the BER performance was better than when the frequency averaging was considered. The improvement compared to the performance of the LS estimator was due to the dynamic update of the channel estimation, which is performed for each symbol, instead of maintaining the initial estimate for all the data frame.

In addition to the BER curves in Figure 11, Table 8 shows the required Eb/No levels to attain 10\%, 1\%, and $0.1 \%$ BER in low mobility conditions over the three described propagation scenarios by considering frame lengths ranging from 16 to 128 OFDM symbols. From Table 8, we can observe that the performance of the modified STA technique decreased as the frame length increased. However, significant performance improvement was observed compared to that obtained by employing the original STA technique (cf. Table 6). Such improvement allowed yielding BER levels of 1\% when the Eb/No regimes were greater than $19.05 \mathrm{~dB}$ for a frame length of 64 , and greater than $22.46 \mathrm{~dB}$ for a frame length of 128 . For these frame lengths, the original STA technique did not attain such BER performance. Furthermore, this modification also allowed reaching $0.1 \%$ BER levels for high $\mathrm{Eb} /$ No regimes, which is an improvement with respect to the performance of the LS estimator in the same simulation conditions. This improvement is because the modified STA technique performes a dynamical estimation that trackes the channel variations from symbol to symbol. This action is not performed by the LS technique, whose initial estimate is used to equalize all the symbols in the frame. Although requiring higher Eb/No levels, the proposed modification enabled attaining BER performances that were not reachable when employing the LS estimator. In addition, the modified STA scheme overcame the limitations of its original version in non-WSSUS channels by removing the spectral averaging operations.

Table 8. Required Eb/No levels to attain $10 \%, 1 \%$, and $0.1 \%$ BER over Scenarios I, II, and III by employing the STA modification proposal for different frame lengths with initial speeds of $v_{T}=v_{R}=40 \mathrm{~km} / \mathrm{h}$ and acceleration components of $a_{T}=a_{R}=20 \mathrm{~m} / \mathrm{s}^{2}$ (NA-not achieved).

\begin{tabular}{ccccccccccccccc}
\hline & \multicolumn{3}{c}{ Scenario I } & \multicolumn{1}{c}{ Scenario II } & \multicolumn{3}{c}{ Scenario III } \\
\hline $\begin{array}{c}\text { Frame length } \\
F\end{array}$ & & 16 & 32 & 64 & 128 & 16 & 32 & 64 & 128 & 16 & 32 & 64 & \multirow{2}{*}{128} \\
\hline Estimator & BER & & & \multicolumn{1}{c}{ Required Eb/No (dB) } \\
\hline Mod. STA $(\alpha=2)$ & $10^{-1}$ & 4.85 & 6.01 & 7.18 & 8.75 & 4.85 & 6.05 & 7.29 & 8.68 & 4.81 & 6.11 & 7.35 & 8.7 \\
\hline Mod. STA $(\alpha=2)$ & $10^{-2}$ & 15.88 & 17.21 & 18.67 & 22.46 & 15.93 & 17.15 & 19.05 & 21.83 & 15.76 & 17.36 & 18.97 & 21.99 \\
\hline Mod. STA $(\alpha=2)$ & $10^{-3}$ & 29.96 & 31.04 & 38.9 & NA & 27.06 & 30.68 & 38.66 & NA & 27.1 & 30.96 & 39.2 & NA \\
\hline
\end{tabular}




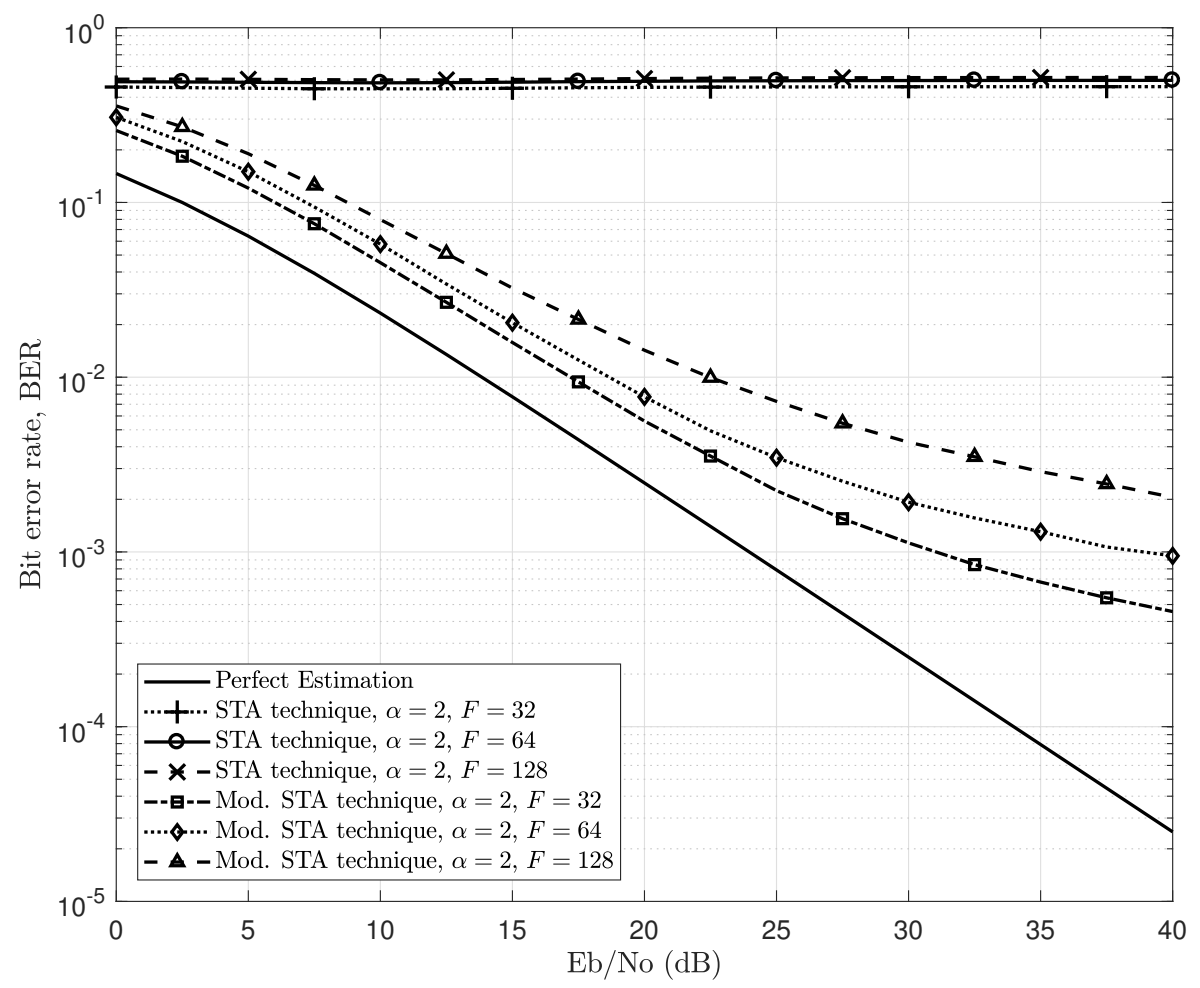

Figure 11. System performance by considering the modified STA technique with different frame lengths, initial speeds of $v_{T}=v_{R}=40 \mathrm{~km} / \mathrm{h}$, and acceleration components of $a_{T}=a_{R}=20 \mathrm{~m} / \mathrm{s}^{2}$ for Scenario I.

\section{Conclusions}

This article presents a simulator that allows reproducing non-WSSUS multipath fading channels under arbitrary isotropic or non-isotropic scattering conditions. Furthermore, the proposed simulator allows velocity variations and non-linear trajectories of the MSs. This simulator is based on the combination of the SOC method and the Monte Carlo principle, and it can be adapted to the multicarrier transmission parameters of the schemes specified in the IEEE 802.11p and LTE-V2X standards. Therefore, the channel simulator can be used to evaluate the performance of V2X communication systems based on those standards for different scenarios of interest. In this paper, the proposed channel simulator was applied to the BER performance analysis of two channel estimation techniques for DSRC transceivers based on the IEEE 802.11p standard, namely the LS estimator and the STA estimator. Our proposal was evaluated in three propagating scenarios for road safety applications. The simulation results showed that the performance of the LS estimator largely depended on the motion profiles of the MSs, the frame length, and the scattering scenario. This is due to the underlying channel estimation procedure since the estimate is computed using only the two initial training symbols and is maintained constant until the end of the frame. Therefore, the estimation can lose its validity over time with the channel variations. An important observation of the LS estimator is that neither the non-stationarities of the channel, nor the acceleration of the MSs have a significant impact on its BER performance. Regarding the STA technique, the non-stationarities of the channel affect its performance, in addition to the losses caused by high mobility conditions, frame length, or scattering scenario. Therefore, in this work, we propose a variant of the STA technique as an alternative method for the estimation of non-WSSUS channels in DSRC systems. The modified STA scheme improves its estimation performance remarkably for Eb/No levels greater than $15 \mathrm{~dB}$, while for smaller values, its performance worsens since the dynamic updates are affected by the high level of noise. This variant of the STA technique is an option that must be analyzed in greater detail for its possible implementation in DSRC systems. In addition, new estimation techniques are necessary to 
deal with the inherent non-stationarities that are found in vehicular channels. In this sense, the simulation framework presented here can be used as a reference for the evaluation of new transmission schemes for a V2X communication system under arbitrary scattering and mobility conditions.

Author Contributions: Conceptualization, J.J.J.-R., C.A.G.-V., C.A.G., J.M.L.-R., D.U.C.-D., and R.V.; methodology, J.J.J.-R., C.A.G.-V., and C.A.G.; software, J.J.J.-R. and C.A.G.-V.; validation, C.A.G., J.M.L.-R., D.U.C.-D., and R.V.; formal analysis, J.J.J.-R., C.A.G.-V., C.A.G., J.M.L.-R., D.U.C.-D., and R.V.; investigation, J.J.J.-R. and C.A.G.-V.; resources, J.J.J.-R., C.A.G., J.M.L.-R., D.U.C.-D., and R.V.; writing, original draft preparation, J.J.J.-R., C.A.G.-V., and C.A.G.; writing, review and editing, J.M.L.-R., D.U.C.-D., and R.V.; supervision, C.A.G.; project administration, J.J.J.-R., C.A.G., J.M.L.-R., and D.U.C.-D.; funding acquisition, J.J.J.-R., C.A.G., J.M.L.-R., D.U.C.-D., and R.V. All authors read and agreed to the published version of the manuscript.

Funding: This research was funded by CONACYT Grant Number 254637 and by PRODEP Grant Number 511-6/2019-8024 (Folio:UASLP-PTC-642).

Conflicts of Interest: The authors declare no conflict of interest.

\section{Abbreviations}

The following abbreviations are used in this manuscript:

3GPP Third-Generation Partnership Project

5G fifth-generation

AOA angle of arrival

AOD angle of departure

AWGN additive white Gaussian noise

BER bit error rate

CIR channel impulse response

C-V2X cellular-based V2X

DMRS demodulation reference signal

DSRC dedicated short-range communications

D2D device-to-device

F2M fixed-to-mobile

GI guard interval

IEEE Institute of Electrical and Electronics Engineers

i.i.d. independent and identically distributed

IO interfering object

ITS intelligent transportation systems

LS least squares

LTE Long-Term Evolution

LTE-V2X LTE-based V2X

LTS long training symbols

MSs mobile stations

OFDM orthogonal frequency division multiplexing

$\mathrm{RB}$ resource blocks

SC-FDMA single carrier frequency division multiple access

SOC sum-of-cisoids

STA spectral temporal averaging

STS short training symbols

TF time and frequency

V2X vehicle-to-everything

V2I vehicle-to-infrastructure

V2N vehicle-to-network

V2P vehicle-to-pedestrian

V2V vehicle-to-vehicle

WLAN wireless local area network

WSS wide sense stationary

WSSUS wide sense stationary uncorrelated scattering 


\section{References}

1. Dimitrakopoulos, G.; Demestichas, P. Intelligent Transportation Systems: Systems Based on Cognitive Networking Principles and Management Functionality. IEEE Veh. Technol. Mag. 2010, 5, 77-84. [CrossRef]

2. Aoyagi, S.; Le, Y.; Shimizu, T.; Takahashi, K. Mobile Application to Provide Traffic Congestion Estimates and Tourism Spots to Promote Additional Stopovers. Future Internet 2020, 12, 83. [CrossRef]

3. Papadimitratos, P.; Fortelle, A.L.; Evenssen, K.; Brignolo, R.; Cosenza, S. Vehicular Communication Systems: Enabling Technologies, Applications, and Future Outlook on Intelligent Transportation. IEEE Commun. Mag. 2009, 47, 84-95. [CrossRef]

4. Kenney, J.B. Dedicated Short-Range Communications (DSRC) Standards in the United States. Proc. IEEE 2011, 99, 1162-1182. [CrossRef]

5. Karagiannis, G.; Altintas, O.; Ekici, E.; Heijenk, G.; Jarupan, B.; Lin, K.; Weil, T. Vehicular Networking: A Survey and Tutorial on Requirements, Architectures, Challenges, Standards and Solutions. IEEE Commun. Surv. Tutor. 2011, 13, 584-616. [CrossRef]

6. Uzcátegui, R.A.; Acosta-Marum, G. WAVE: A Tutorial. IEEE Commun. Mag. 2009, 47, 126-133. [CrossRef]

7. Seo, H.; Lee, K.; Yasukawa, S.; Peng, Y.; Sartori, P. LTE Evolution for Vehicle-to-Everything Services. IEEE Commun. Mag. 2016, 54, 22-28. [CrossRef]

8. Chen, S.; Hu, J.; Shi, Y.; Peng, Y.; Fang, J.; Zhao, R.; Zhao, L. Vehicle-to-Everything (V2X) Services Supported by LTE-Based Systems and 5G. IEEE Commun. Stand. Mag. 2017, 1, 70-76. [CrossRef]

9. Bazzi, A.; Cecchini, G.; Menarini, M.; Masini, B.; Zanella, A. Survey and Perspectives of Vehicular Wi-Fi versus Sidelink Cellular-V2X in the 5G Era. Future Internet 2019, 11, 122. [CrossRef]

10. Soto, I.; Jimenez, F.; Calderon, M.; Naranjo, J.E.; Anaya, J.J. Reducing Unnecessary Alerts in Pedestrian Protection Systems Based on P2V Communications. Electronics 2019, 8, 1-26.

11. IEEE. IEEE Std. 802.11-2010 (Revision of IEEE Std 802.11-1999), Wireless LAN Medium Access Control (MAC) and Physical Layer (PHY) Specifications. Amendment 6: Wireless Access in Vehicular Environments, Part 11 ed.; IEEE: New York, NY, USA, 2010; pp. 1-2793.

12. Wang, X.; Mao, S.; Gong, M. An Overview of 3GPP Cellular Vehicle-to-Everything Standards. Get Mob. Mob. Comput. Commun. 2017, 21, 19-25. [CrossRef]

13. Khan, U.A.; Lee, S. Three-Dimensional Resource Allocation in D2D-Based V2V Communication. Electronics 2019, 8, 1-15.

14. 3rd Generation Partnership Project. 3GPP TR 21.914 V14.0.0 (2018-05) 3rd Generation Partnership Project; Technical Specification Group Services and System Aspects; Release 14 Description; Summary of Rel-14 Work Items (Release 14); 3GPP: Valbonne, France, 2018.

15. 3rd Generation Partnership Project. 3GPP TR 21.916 V0.4.0 (2020-03) 3rd Generation Partnership Project; Technical Specification Group Services and System Aspects; Release 16 Description; Summary of Rel-16 Work Items (Release 16); 3GPP: Valbonne, France, 2019.

16. Viriyasitavat, W.; Boban, M.; Tsai, H.; Vasilakos, A. Vehicular Communications: Survey and Challenges of Channel and Propagation Models. IEEE Veh. Technol. Mag. 2015, 10, 55-66. [CrossRef]

17. Atallah, R.F.; Khabbaz, M.J.; Assi, C.M. Vehicular Networking: A Survey on Spectrum Access Technologies and Persisting Challenges. Elsevier Veh. Commun. 2015, 2, 125-149. [CrossRef]

18. Khan, U.A.; Lee, S. Multi-Layer Problems and Solutions in VANETs: A Review. Electronics 2019, 8, 1-19.

19. Bakhshi, G.; Saadat, R.; Shahtalebi, K. Modeling and Simulation of MIMO Mobile-to-Mobile Wireless Fading Channels. Int. J. Antennas Propag. 2012, 2012, 1-13. [CrossRef]

20. Qiuming, Z.; Xinglin, L.; Ning, L.; Xiaomin, C. An Improved Sum-of-Sinusoids Channel Simulator Based on Brownian Motion. In Proceedings of the 2014 International Symposium on Antennas and Propagation Conference, Kaohsiung, Taiwan, 2-5 December 2014; pp. 407-408.

21. Ma, Y.; Pätzold, M. A Wideband One-Ring MIMO Channel Model Under Non-Isotropic Scattering Conditions. In Proceedings of the VTC Spring 2008-IEEE Vehicular Technology Conference, Marina Bay, Singapore, 11-14 May 2008; pp. 424-429.

22. Papoulis, A.; Pillai, S.U. Probability, Random Variables and Stochastic Processes, 4th ed.; McGraw-Hill: New York, NY, USA, 2002. 
23. Bernadó, L.; Zemen, T.; Tufvesson, F.; Molisch, A.F.; Mecklenbrauker, C.F. The (In-)validity of the WSSUS Assumption in Vehicular Radio Channels. In Proceedings of the 2012 IEEE 23rd International Symposium on Personal, Indoor and Mobile Radio Communications-(PIMRC), Sydney, Australia, 9-12 September 2012; pp. 1757-1762.

24. Bernadó, L.; Zemen, T.; Tufvesson, F.; Molisch, A.F.; Mecklenbrauker, C.F. Delay and Doppler Spreads of Nonstationary Vehicular Channels for Safety-Relevant Scenarios. IEEE Trans. Veh. Technol. 2014, 63, 82-93. [CrossRef]

25. Mecklenbrauker, C.; Molisch, A.; Karedal, J.; Tufvesson, F.; Paier, A.; Bernardo, L.; Zemen, T.; Klemp, O.; Czink, N. Vehicular Channel Characterization and its Implications for Wireless System Design and performance. Proc. IEEE 2011, 99, 1189-1212. [CrossRef]

26. Narrainen, J.; Besnier, P.; Gatsinzi Ibambe, M. A Geometry-Based Stochastic Approach to Emulate V2V Communications' Main Propagation Channel Metrics. Int. J. Microw. Wirel. Technol. 2016, 8, 455-461. [CrossRef]

27. Ivan, I.; Besnier, P.; Bunion, X.; Danvic, L.L.; Drissi, M. On the Simulation of Weibull Fading for V2X Communications. In Proceedings of the 2011 11th International Conference on ITS Telecommunications, St. Petersburg, Russia, 23-25 August 2011; pp. 86-91.

28. Li, Y.; Ai, B.; Cheng, X.; Lin, S.; Zhong, Z. A TDL Based Non-WSSUS Vehicle-to-Vehicle Channel Model. Int. J. Antennas Propag. 2013, 2013, 1-8. [CrossRef]

29. Li, Y.; Ai, B.; Michelson, D.G.; Lin, S.; Wang, Q.; Zhong, Z. A Method for Generating Correlated Taps in Stochastic Vehicle-to-Vehicle Channel Models. In Proceedings of the 2015 IEEE 81st Vehicular Technology Conference (VTC Spring), Glasgow, Scotland, 1-14 May 2015; pp. 1-5.

30. Jeruchim, M.C.; Balaban, P.; Shanmugan, K.S. Simulation of Communication Systems, 2nd ed.; Springer: New York, NY, USA, 2000.

31. Gutierrez, C.A.; Patzold, M.; Dahech, W.; Youssef, N. A Non-WSSUS Mobile-to-Mobile Channel Model Assuming Velocity Variations of the Mobile Stations. In Proceedings of the 2017 IEEE Wireless Communications and Networking Conference(WCNC), San Francisco, CA, USA, 19-22 March 2017; pp. 1-6.

32. Fernandez, J.A.; Borries, K.; Cheng, L.; Kumar, B.V.K.V.; Stancil, D.D.; Bai, F. Performance of the 802.11p Physical Layer in Vehicle-to-Vehicle Environments. IEEE Trans. Veh. Technol. 2012, 61, 3-14. [CrossRef]

33. Vatalaro, F.; Forcella, A. Doppler spectrum in mobile-to-mobile communications in the presence of three-dimensional multipath scattering. IEEE Trans. Veh. Technol. 1997, 46, 213-219. [CrossRef]

34. Patel, C.; Stuber, G.; Pratt, T. Simulation of Rayleigh-faded mobile-to-mobile communication channels. IEEE Trans. Commun. 2005, 53, 1876-1884. [CrossRef]

35. Pätzold, M.; Hogstad, B.O.; Youssef, N. Modeling, analysis, and simulation of MIMO mobile-to-mobile fading channels. IEEE Trans. Wirel. Commun. 2008, 7, 510-520. [CrossRef]

36. Zajić, A.G.; Stüber, G.L. Space-Time Correlated Mobile-to-Mobile Channels: Modelling and Simulation. IEEE Trans. Veh. Technol. 2008, 57, 715-726. [CrossRef]

37. Zajić, A.G.; Stüber, G.L.; Pratt, T.G.; Nguyen, T. Wideband MIMO Mobile-to-Mobile Channels: Geometry-Based Statistical Modeling With Experimental Verification. IEEE Trans. Veh. Technol. 2009, 58, 517-534. [CrossRef]

38. Zajić, A.G.; Stüber, G.L. Three-Dimensional Modeling and Simulation of Wideband MIMO Mobile-to-Mobile Channels. IEEE Trans. Wirel. Commun. 2009, 8, 1260-1275. [CrossRef]

39. Cheng, X.; Yao, Q.; Wen, M.; Wang, C.X.; Song, L.Y.; Jiao, B.L. Wideband Channel Modeling and Intercarrier Interference Cancellation for Vehicle-to-Vehicle Communication Systems. IEEE J. Sel. Areas Commun. 2013, 31, 434-448. [CrossRef]

40. Walter, M.; Shutin, D.; Fiebig, U.C. Delay-Dependent Doppler Probability Density Functions for Vehicle-to-Vehicle Scatter Channels. IEEE Trans. Antennas Propag. 2014, 62, 2238-2249. [CrossRef]

41. Karedal, J.; Tufvesson, F.; Czink, N.; Paier, A.; Dumard, C.; Zemen, T.; Mecklenbräuker, C.; Molisch, A.F. A Geometry-Based Stochastic MIMO Model for Vehicle-to-Vehicle Communications. IEEE Trans. Wirel. Commun. 2009, 8, 3646-3657. [CrossRef]

42. Gutiérrez, C.A.; Gutiérrez-Mena, J.T.; Luna-Rivera, J.M.; Campos-Delgado, D.U.; Velázquez, R.; Pätzold, M. Geometry-Based Statistical Modeling of Non-WSSUS Mobile-to-Mobile Rayleigh Fading Channels. IEEE Trans. Veh. Technol. 2017, 67, 362-377. [CrossRef] 
43. Gutiérrez, C.A.; Jaime-Rodríguez, J.J.; Luna-Rivera, J.M.; Campos-Delgado, D.U.; Vázquez-Castillo, J. Modeling of non-WSSUS Double-Rayleigh Fading Channels for Vehicular Communications. Wirel. Commun. Mob. Comput. 2017, 2017, 1-13. [CrossRef]

44. Yuan, Y.; Wang, C.X.; He, Y.; Alwakeel, M.M.; Aggoune, E.H.M. 3D wideband non-stationary geometry-based stochastic models for non-isotropic MIMO vehicle-to-vehicle channels. IEEE Trans. Wirel. Commun. 2015, 14, 6883-6895. [CrossRef]

45. Iqbal, R.; Abhayapala, T.D. Impact of mobile acceleration on the statistics of Rayleigh fading channel. In Proceedings of the Australian Communication Theory Workshop (AusCTW), Adelaide, Australia, 5-7 February 2007; pp. 122-128.

46. Pätzold, M.; Borhani, A. A non-stationary multipath fading channel model incorporating the effect of velocity variations of the mobile station. In Proceedings of the WCNC: Wireless Communications and Networking Conference, Honolulu, HI, USA, 3-6 February 2014; pp. 194-199.

47. Dahech, W.; Pätzold, M.; Gutiérrez, C.A.; Youssef, N. A Non-Stationary Mobile-to-Mobile Channel Model Allowing for Velocity and Trajectory Variations of the Mobile Stations. IEEE Trans. Wirel. Commun. 2017, 16, 1987-2000. [CrossRef]

48. Jiang, K.; Chen, X.; Zhu, Q.; Chen, L.; Xu, D.; Chen, B. A Novel Simulation Model for Nonstationary Rice Fading Channels. Wirel. Commun. Mob. Comput. 2018, 2018, 1-9. [CrossRef]

49. Imbert, S.; Leturc, X.; LeMartret, C.J. On the simulation of correlated mobile-to-mobile fading channels for time-varying velocities. In Proceedings of the 2018 International Conference on Military Communications and Information Systems (ICMCIS 2018), Mazowieckie, Poland, 22-23 May 2018; pp. 1-8.

50. Gutiérrez, C.A.; Pätzold, M.; Ortega-Silva, N.; Azurdia-Meza, C.A.; Maciel-Barbosa, F.M. Doppler Shift Characterization of Wideband Mobile Radio Channels. IEEE Trans. Veh. Technol. 2019, 68, 12375-12380. [CrossRef]

51. von Mises, R. Über die 'Ganzzahligkeit' der Atomgewichte und verwandte Fragen. Phys. Z. 1918, 19, 490-500.

52. Pätzold, M. Mobile Radio Channels, 2nd ed.; John Wiley and Sons: Chichester, UK, 2011.

53. Gutiérrez, C.A.; Pätzold, M. The Generalized Method of Equal Areas for the Design of Sum-of-Cisoids Simulators for Mobile Rayleigh Fading Channels with Arbitrary Doppler Spectra. Wirel. Commun. Mob. Comput. 2011, 13, 951-966. [CrossRef]

54. Gutiérrez, C.A.; Pätzold, M. The Design of Sum-of-Cisoids Rayleigh Fading Channel Simulators Assuming non-Isotropic Scattering Conditions. IEEE Trans. Wirel. Commun. 2010, 9, 1308-1314. [CrossRef]

55. Hogstad, B.O.; Gutiérrez, C.A.; Pätzold, M.; Crespo, P.M. Classes of Sum-of-Cisoids Processes and Their Statistics for the Modeling and Simulation of Mobile Fading Channels. EURASIP J. Wirel. Commun. Netw. 2013, 2013, 125. [CrossRef]

56. Jaime-Rodríguez, J.; Gutiérrez, C.A.; Luna-Rivera, M.; Campos-Delgado, D.U.; Velazquez, R. Comparative Performance Analysis of Two Channel Estimation Techniques for DSRC Systems Based on the IEEE 802.11p Standard. In Proceedings of the 34th IEEE Convention of Central America and Panama (CONCAPAN XXXIV), Panama City, Panama, 12-14 November 2014; pp. 1-6. (In Spanish)

57. Jaime-Rodríguez, J.J.; Gómez-Vega, C.A.; Gutiérrez, C.A.; Luna-Rivera, J.M.; Campos-Delgado, D.U.; Velázquez, R. A Monte Carlo Simulator of non-WSSUS Rayleigh Fading Channels for Vehicular Communications. In Proceedings of the 2018 IEEE 38th Central America and Panama Convention (CONCAPAN XXXVIII), San Salvador, El Salvador, 7-9 November 2018; pp. 1-6.

58. Ortega, N.M.; Azurdia-Meza, C.A.; Gutierrez, C.A.; Gómez-Vega, C.A. Second Order Statistics and BER Performance Analysis of a non-WSSUS V2X Channel Model that Considers Velocity Variations. In Proceedings of the 2019 IEEE Latin-American Conference on Communications (LATINCOM), Salvador, Brazil, 11-13 November 2019; pp. 1-6.

59. Gómez-Vega, C.A.; Jaime-Rodríguez, J.J.; Gutiérrez, C.A.; Velázquez, R. Bit error rate performance analysis of vehicular communication systems considering velocity variations of the mobile stations. In Proceedings of the 2017 IEEE 37th Central America and Panama Convention (CONCAPAN XXXVII), Managua, Nicaragua, 15-17 November 2017; pp. 1-6.

(C) 2020 by the authors. Licensee MDPI, Basel, Switzerland. This article is an open access article distributed under the terms and conditions of the Creative Commons Attribution (CC BY) license (http:/ / creativecommons.org/licenses/by/4.0/). 